\title{
Axi-Symmetrical Transient Temperature Fields and Quasi-Static Thermal Stresses Initiated by a Laser Pulse in a Homogeneous Massive Body
}

\author{
Aleksander Yevtushenko ${ }^{1}$, Kazimierz Rozniakowski ${ }^{2}$ \\ and Malgorzata Rozniakowska-Klosinska ${ }^{3}$ \\ ${ }^{1}$ Bialystok University of Technology \\ Faculty of Mechanical Engineering \\ ${ }^{2}$ Technical University of Lodz Faculty of Technical Physics \\ Information Technology and Applied Mathematics \\ ${ }^{3}$ Technical University of Lodz
}

Poland

\section{Introduction}

In the present chapter the model of a semi-infinite massive body which is heated through the outer surface by the precised heat flux, is being under study. This heat flux has the intensity directly proportional to the equivalent laser irradiation intensity. Heating of materials due to its surface irradiation by the high-power energy fluxes, which takes place during working of the laser systems, can be modelled in a specific conditions as the divided surface heat source of defined power density or heat flux of defined intensity (Rykalin et al., 1975).

Laser systems are an unusual source of electromagnetic irradiation of unique properties. These properties differ essentially from the relevant characteristics of irradiation generated by traditional sources, natural and artificial one. Laser irradiation has specific, distinguishing features: high level of spatial and time coherence, high level of monochromaticity, low divergence, high spectral intensity and continuous or impulse emission process. High level of spatial coherence gives possibility to focusing laser irradiation on the surfaces of a few to several dozens squared micrometers in a size, which correspond to very high values of power intensity even $10^{8}-10^{12} \mathrm{~W} / \mathrm{m}^{2}\left(10^{4}-10^{18} \mathrm{~J} / \mathrm{m}^{2}\right.$ or $10^{23}$ fotons $/ \mathrm{cm}^{2}$ ). Effectivity of local surface heating mentioned above depends on: laser pulse duration, laser pulse structure (shape) and on irradiation intensity distribution. Three specific laser pulse structures are usually under consideration: rectangular-shape pulse, triangular-shape pulse and pulse shape approximated by some defined function. Likewise to the laser pulse structure, the spatial pulse structure (distribution of laser irradiation in a plane normal to the beam axis) is also complex and challenging for precised analytical description. In approximation the spatial distribution of laser irradiation can be described by the following relations: gaussian distribution (takes place during the working of laser beam in the single-mode regime), mixed (multi-modal) or uniform distribution. In addition, laser heat source shape can be changed by the electromagnetic or optical methods. Hence, the 
optimization of the source shape problem appears on the basis of various optimisation criteria as well as the minimal losses on apparatus criterion.

In the former industrial practice, mainly the gaussian or uniform intensity distribution were applied. Various in nature thermal effects are present during industrial laser materials processing such as: laser hardening, laser surface modifications of metals and alloys. Nowadays the most significant role in the technological operations plays such formed laser beam which maximum power is achieved not in the centre but close to the edge of the heated zone (Hector \& Hetnarski, 1996). That is why, in the emerging process of the new effective laser technologies, it is strongly reasonable to determine the analytical solutions and to conduct numerical analysis for the boundary value problem of transient heat conduction and quasi-static thermal stresses, which are crucial in calculations of:

- the effective absorption coefficient,

- the specific time point when surface melting occurs due to laser beam heating,

- the heating velocity and cooling,

- the controlled laser thermo cracking process (Lauriello \& Chen, 1973; Yevtushenko et al., 1997),

- $\quad$ the other features of initiated temperature and thermal stresses fields.

\section{Influence of intensity spatial distribution of laser beam on a temperature field in the irradiated massive body (semi-infinite)}

\subsection{Problem statement}

Laser irradiation interaction of $10^{4} \div 10^{8} \mathrm{~W} / \mathrm{m}^{2}$ power intensity on metals is equivalent to heating them by heat flux of defined intensity (Rykalin et al., 1975). If the following conditions are fulfilled:

- the power intensity generated by the laser is not sufficient to melt and evaporate the superficial layer,

- the losses because of heat emission and convection from a surface body are negligible

- the thermo-physical properties do not depend on temperature,

then the axisymmetrical boundary value problem of heat conduction for semi-infinite body in cylindrical coordinates system $(r, z)$ with the beginning in the centre of heated surface, can be considered in the form:

$$
\begin{gathered}
\frac{\partial^{2} T}{\partial r^{2}}+\frac{1}{r} \frac{\partial T}{\partial r}+\frac{\partial^{2} T}{\partial z^{2}}=\frac{1}{k} \frac{\partial T}{\partial t}, r \geq 0, z>0, t>0, \\
T(r, z, 0)=0, r \geq 0, z \geq 0, \\
K \frac{\partial T}{\partial z}=-A q(r) H\left(t_{s}-t\right), r \geq 0, z=0, t>0, \\
T(r, z, t) \rightarrow 0, \sqrt{r^{2}+z^{2}} \rightarrow \infty, t>0 .
\end{gathered}
$$

The uniform distribution of heat flux intensity in a circle of $a$ radius, can be described by the formula: 


$$
q(r)=q_{0} H(a-r), r \geq 0
$$

and for the mixed distribution - multimodal one, the heat flux intensity can be expressed by (Hector \& Hetnarski, 1996):

$$
q(r)=q_{f}\left[f+(1-f) K_{c} r^{2}\right] e^{-K_{c} r^{2}}, r \geq 0,
$$

where $K_{c}$ - concentration coefficient, $q_{f}$ - characteristic value of heat flux intensity $q$, $0 \leq f \leq 1$ - parameter, which characterized the irradiation intensity distribution in a plane normal to the laser beam axis. For $f=1$ the normal (gaussian) distribution and for $f=0$ doughnut - toroidal distribution, is obtained.

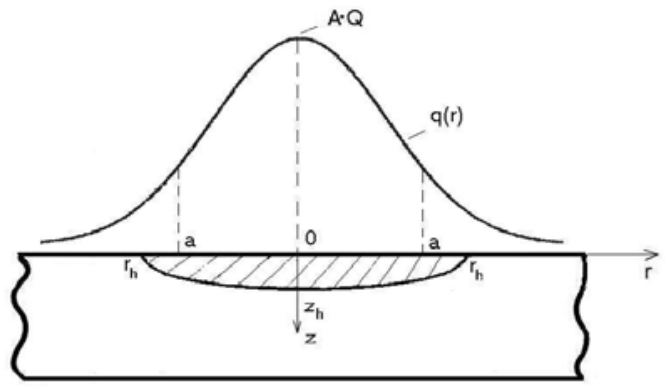

Fig. 1. Laser irradiation heating model and area shape visualization of phase transition for metals

Both distributions of laser irradiation intensity (5) and (6) are related by the following concentration coefficient (Rykalin et al., 1975)

$$
K_{c}=B_{f} a^{-2} .
$$

The numerical factor $B_{f}$ in the Eq. (7) can be found from the condition below (Hector \& Hetnarski, 1996):

$$
\frac{Q_{f}}{Q}=\left(1-\frac{1}{e}\right) 100 \% \approx 63.2 \%,
$$

where $Q$ - total irradiation power, $Q_{f}$ - irradiation power which arrives to the circle of $a$ radius and can be easily derived after taking into consideration the distribution (6), consequently these two values can be substituted into Eq. (8). As the result the non-linear equation will be received for $B_{f}$ in the form:

$$
\left[1+(1-f) B_{f}\right] e^{-B_{f}}=e^{-1} \approx 0.3678 .
$$

A numerical analysis shows that dependence of the $B_{f}$ roots of Eq. (11) with respect to the $f$ parameter is nearly linear: $B_{f}=B_{0}(1-f)+f$, where $B_{0}=2.1462$ is the value of $B_{f}$ at 
$f=0$. At $f=1$ from Eq. (6) is received the obvious result $B_{f}=1$ (Rykalin et al., 1975). By comparizing the irradiation intensity of uniform distribution (5) with the irradiation intensity of general case distribution (6) it was found

$$
q_{f}=B_{f} q_{0} .
$$

By introducing dimensionless variables and parameters

$$
\rho=\frac{r}{a}, \zeta=\frac{z}{a}, \tau=\frac{k t}{a^{2}}, \tau_{s}=\frac{k t_{s}}{a^{2}}, T_{0}=\frac{q_{0} a}{K}, T^{*}=\frac{T}{T_{0}},
$$

and using relations (7) and (12) the boundary value heat conductivity problem (1)-(4) can be rewritten in the form:

$$
\begin{gathered}
\frac{\partial^{2} T^{*}}{\partial \rho^{2}}+\frac{1}{\rho} \frac{\partial T^{*}}{\partial \rho}+\frac{\partial^{2} T^{*}}{\partial \zeta^{2}}=\frac{\partial T^{*}}{\partial \tau}, \rho \geq 0, \zeta>0, \tau>0, \\
T^{*}(\rho, \zeta, 0)=0, \rho \geq 0, \zeta \geq 0, \\
\frac{\partial T^{*}}{\partial \zeta}=-A q^{*}(\rho) H\left(\tau_{s}-\tau\right), \rho \geq 0, \zeta=0, \tau>0 \\
T^{*}(\rho, \zeta, \tau) \rightarrow 0, \sqrt{\rho^{2}+\zeta^{2}} \rightarrow \infty, \tau>0
\end{gathered}
$$

where

$$
q^{*}(\rho)=B_{f}\left[f+(1-f) B_{f} \rho^{2}\right] e^{-B_{f} \rho^{2}},(\text { see Fig. 2) }
$$

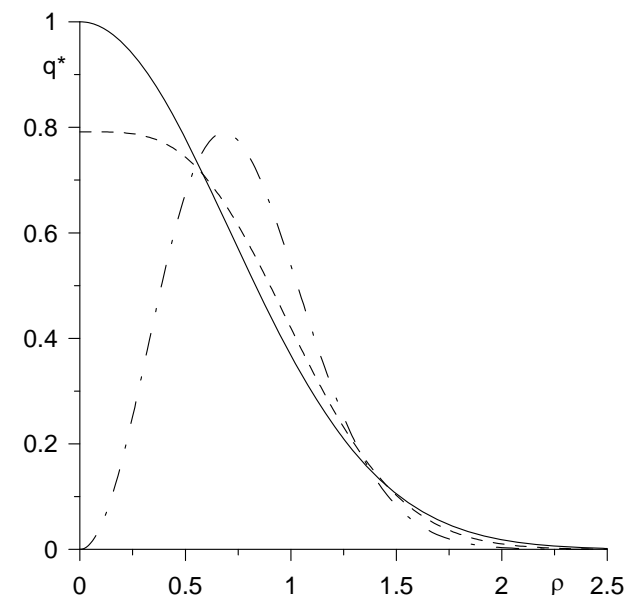

Fig. 2. Laser irradiation distribution - a function $q^{*}(\rho)$ for three different $f$ parameter values (solid line corresponds to the value of $f=1$, dashed line to $f=0.5$, dot-dashed line to $f=0$ ). 


\subsection{Problem solution}

The solution of the boundary value heat conduction problem (14)-(17), which is obtained by applying the integral Hankel transforms with respect to the radial variable $r$ and Laplace transform with respect to time $t$, has the following form:

$$
\begin{gathered}
T^{*}(\rho, \zeta, \tau)=T^{(0) *}(\rho, \zeta, \tau) H(\tau)-T^{(0) *}\left(\rho, \zeta, \tau-\tau_{s}\right) H\left(\tau-\tau_{s}\right), \quad \rho \geq 0, \zeta \geq 0, \tau \geq 0 . \\
T^{(0) *}(\rho, \zeta, \tau)=A \int_{0}^{\infty} \varphi(\xi) \Phi(\xi, \zeta, \tau) J_{0}(\xi \rho) d \xi, \rho \geq 0, \zeta \geq 0, \tau \geq 0
\end{gathered}
$$

where

$$
\varphi(\xi)=\int_{0}^{\infty} \rho q^{*}(\rho) J_{0}(\xi \rho) d \rho=\frac{1}{2}\left[f+(1-f)\left(1-\frac{\xi^{2}}{4 B_{f}}\right)\right] e^{-\frac{\xi^{2}}{4 B_{f}}}, \xi \geq 0
$$

and

$$
\Phi(\xi, \zeta, \tau)=\frac{1}{2}\left[e^{-\xi \zeta} \operatorname{erfc}\left(\frac{\zeta}{2 \sqrt{\tau}}-\xi \sqrt{\tau}\right)-e^{\xi \zeta} \operatorname{erfc}\left(\frac{\zeta}{2 \sqrt{\tau}}+\xi \sqrt{\tau}\right)\right] .
$$

During transient heating of the massive body, the maximum value of temperature on the body surface is achieved at the moment $t=t_{s}\left(\tau=\tau_{s}\right)$ - switching laser system off, whereas inside the body at $t_{h}=t_{s}+\Delta t \quad\left(\tau_{h}=\tau_{s}+\Delta \tau\right.$, where $\left.\Delta \tau=k \Delta t / a^{2}\right)$. When laser system is switched off, then laser heating source is simply cut off - as a consequence the fast cooling of the body takes place.

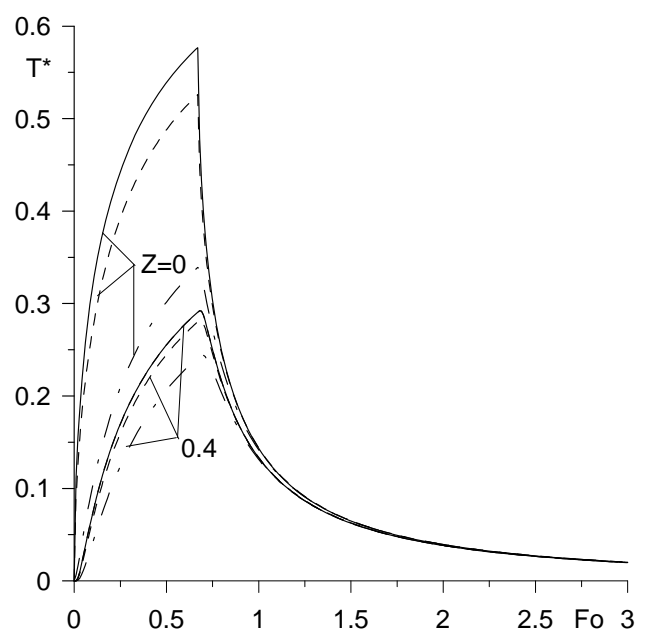

Fig. 3. Evolution of dimensionless temperature $T^{*}=T /\left(A T_{0}\right)$ on the surface in the center of heated zone $(\rho=0, \zeta=0)$ and inside the body $(\rho=0, \zeta=0.4)$ at $\tau_{s}=0.6$ (Yevtushenko et al., 2009). 
Independently from the heat source intensity distribution, the retardation time $\Delta t(\Delta \tau)$ is increasing fast with the distance from the heated surface. For a fixed value of depth from the working surface this retardation time $\Delta t$ decreases with the increase of the $f$ parameter.

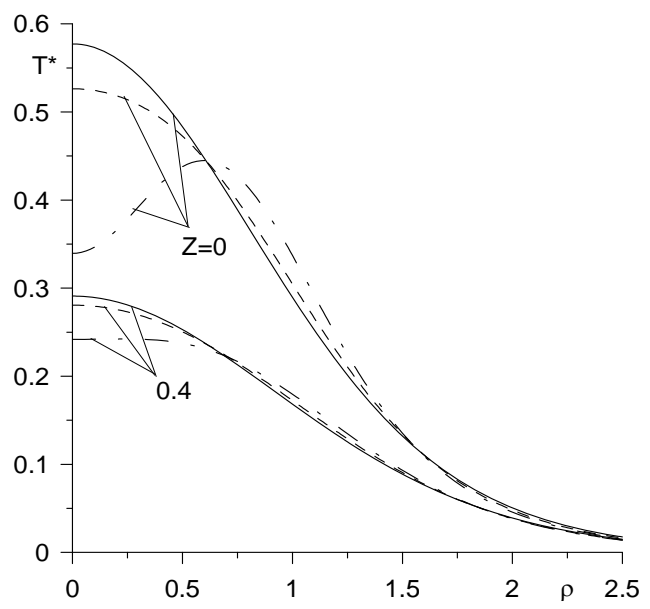

Fig. 4. Evolution of dimensionless temperature $T^{*}$ along $\rho$ variable on the body surface $(\zeta=0)$ and inside the body $(\zeta=0.4)$ at $\tau_{s}=0.6$ (Yevtushenko et al., 2009).

The irradiation intensity distribution in a plane normal to the laser beam axis, see Eq.(18), affects essentially the character of surface temperature distribution. Maximum value of temperature for normal distribution of heat flux intensity is attained at the center of heated zone $r=0$, and for a doughnut distribution is attained near a boundary of the heated zone $r=0.6 a$ (see Fig. 4).

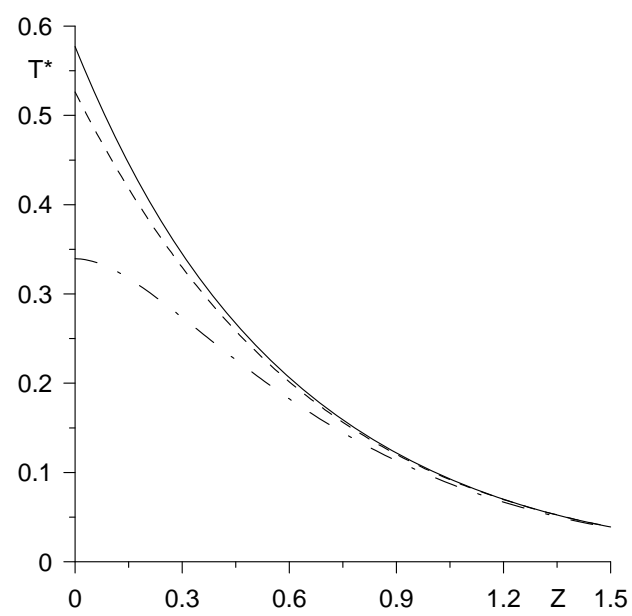

Fig. 5. Evolution of dimensionless temperature $T^{*}$ along symmetry axis $\rho=0$ at $\tau_{s}=0.6$ (Yevtushenko et al., 2009). 
The effective depth of heating (the distance from the boundary body surface for which temperature value equals $5 \%$ of the maximum value of temperature achieved on the surface) is independent on the form of intensity distribution of the incident heat flux and for dimensionless retardation time $\tau_{s}=0.6$ is equal in approximation $1.5 a$ (see Fig. 5). At work (Ashcroft \& Mermin, 1986) was shown that for laser systems working in the continuous regime the above quantity does not exceed the $5 a$ value (Matysiak et al., 1998). This conclusion confirms the results shown in the present chapter thanks to the numerical calculations done with use of formulas (19)-(22) at $\tau_{s} \rightarrow \infty$ and $\tau=100$ (dimensionless time, when stationary temperature is achieved).

\subsection{Special cases of the obtained solution}

At $t_{s} \rightarrow \infty\left(\tau_{s} \rightarrow \infty\right)$ from Eq. (19) is received that $T^{*}(\xi, \zeta, \tau)=T^{(0) *}(\xi, \zeta, \tau)$ and formula (20) corresponds with solution presented in paper (Ready, 1997) for laser operated in the continuous regime and irradiation of semi-infinite surface. If additionally $t \rightarrow \infty(\tau \rightarrow \infty)$, then from Eq. (22) follows that $\Phi(\xi, \zeta, \infty)=e^{-\xi \zeta}$ and then stationary temperature can be derived from the relation:

$$
T^{(0) *}(\rho, \zeta, \infty)=A \int_{0}^{\infty} \varphi(\xi) e^{-\xi \zeta} J_{0}(\xi \rho) d \xi, \rho \geq 0, \zeta \geq 0
$$

By substituting to the Eq. (23) $\zeta=0$, the stationary temperature on surface for semi-infinite surface can be found

$$
T^{(0) *}(\rho, 0, \infty)=A \int_{0}^{\infty} \varphi(\xi) J_{0}(\xi \rho) d \xi, \rho \geq 0 .
$$

Substituting function $\phi(\xi)$ ( 21) under integral and with consideration of integrals:

$$
\int_{0}^{\infty} e^{-\frac{\xi^{2}}{4 B_{f}}} J_{0}(\xi \rho) d \xi \quad \text { and } \quad \int_{0}^{\infty} \xi^{2} e^{-\frac{\xi^{2}}{4 B_{f}}} J_{0}(\xi \rho) d \xi
$$

with use of integrals table (Prudnikov et al., 1998) the solution for stationary temperature on semi-infinite surface was received in the following form:

$$
T(r, 0, \infty)=A \frac{\sqrt{\pi B_{f}}}{2} e^{-\frac{B_{f} \rho^{2}}{2}}\left\{I_{0}\left(\frac{B_{f} \rho^{2}}{2}\right)-\frac{1-f}{2}\left[\left(1-B_{f} \rho^{2}\right) I_{0}\left(\frac{B_{f} \rho^{2}}{2}\right)+B_{f} \rho^{2} I_{1}\left(\frac{B_{f} \rho^{2}}{2}\right)\right]\right\} .
$$

In similar way the distribution of the stationary temperature along axis $\rho=0$ from the (23) solution was found:

$$
T^{(0) *}(0, \zeta, \infty)=A\left\{\frac{\zeta}{2}(1-f)+\left[\frac{1}{2}(1+f)-(1-f) \zeta^{2}\right] \frac{\sqrt{\pi B_{f}}}{2} e^{B_{f} \zeta^{2}} \operatorname{erfc}\left(\sqrt{B_{f}} \zeta\right)\right\} .
$$


From relations (27) and (28) follows, that stationary temperature on the body surface, in the center of heated zone, reaches the value:

$$
T^{(0) *}(0,0, \infty)=A \frac{(1+f)}{4} \sqrt{\pi B_{f}}
$$

For normal distribution of irradiation intensity $\left(f=1, B_{f}=1\right)$ from Eq. (29) the following result is obtained (Bardybahin \& Czubarov, 1996) :

$$
T^{(0) *}(0,0, \infty)=A \frac{\sqrt{\pi}}{2} \approx 0.8862 A
$$

and for doughnut mode structure distribution $\left(f=0, B_{f}=2.1462\right)$

$$
T^{(0) *}(0,0, \infty)=A \frac{\sqrt{\pi B_{f}}}{2} \approx 0.6492 A .
$$

It should be underlined that maximum of stationary temperature is achieved not for gaussian irradiation intensity distribution in a plane normal to the laser beam axis $(f=1)$, but for "almost gaussian" distribution $(f=0.93)$, when $T^{(0) *}(0,0, \infty) \approx 0.8889 A$.

\subsection{Time point determination when surface melting occurs due to laser beam heating}

On the basis of achieved solutions (19)-(23), the time point determination when surface melting occurs due to laser beam heating, can be done. It is known that for uniform Eq. (5) and normal Eq. (6) (for $f=1$ ) distribution of laser heat flux intensity, the maximum temperature is achieved on the surface in the centre of the heated zone. For laser systems working in the continuous generation regime, from solutions (20)-(23) at $t_{s} \rightarrow \infty\left(\tau_{s} \rightarrow \infty\right)$ is derived in general

$$
T^{(0) *}(0,0, \tau)=A \int_{0}^{\infty} \varphi(\xi) \operatorname{erf}(\xi \sqrt{\tau}) d \xi,
$$

In case of uniform distribution (5) the function $\varphi(\xi)=J_{1}(\xi) / \xi$ (Matysiak et al., 1998) and then (32) formula becomes

$$
T^{(0)^{*}}(0,0, \tau)=\int_{0}^{\infty} \frac{J_{1}(\xi)}{\xi} \operatorname{erf}(\xi \sqrt{\tau}) d \xi .
$$

By differentiation of (33) solution along $\beta=\sqrt{\tau}$ variable, the following is found (Prudnikov et al., 1998):

$$
\frac{d T^{(0)^{*}}}{d \beta}=\frac{2}{\sqrt{\pi}} A \int_{0}^{\infty} J_{1}(\xi) e^{-\beta^{2} \xi^{2}} d \xi=\beta^{-1} e^{-\frac{1}{8 \beta^{2}}} I_{1 / 2}\left(\frac{1}{8 \beta^{2}}\right) .
$$

According to work (Abramowitz \& Stegun, 1979): 


$$
I_{1 / 2}(x)=\sqrt{\frac{2}{\pi x}} \operatorname{sh} x
$$

then (34) formula can be rewritten in the form

$$
\frac{d T^{(0)^{*}}}{d \beta}=\frac{2 A}{\sqrt{\pi}}\left(1-e^{-\frac{1}{4 \beta^{2}}}\right)
$$

By integration (36) formula along $\beta$ variable with condition consideration $T^{(0)^{*}}(0,0,0)=0$, the formula of dimensionless temperature evolution in the centre of the heated zone $(\rho=0, \zeta=0)$ is derived:

$$
T^{(0)^{*}}(0,0, \tau)=A\left[2 \sqrt{\frac{\tau}{\pi}}\left(1-e^{-\frac{1}{4 \tau}}\right)+\operatorname{erfc}\left(\frac{1}{2 \sqrt{\tau}}\right)\right] .
$$

The respective formula for gaussian (normal distribution) laser heat flux intensity got the form (Aulyn et al., 2002):

$$
T^{(0)^{*}}(0,0, \tau)=A\left[\frac{\sqrt{\pi}}{2}-\frac{1}{\sqrt{\pi}} \operatorname{arctg} \frac{1}{2 \sqrt{\tau}}\right]
$$

Dimensionless temperatures $T^{(0)^{*}}(0,0, \tau),(37)$ and (38), increase in monotone mode with increase of irradiation time and reach the asymptotes respectively: 1 and $\sqrt{\pi} / 2=0.8862$ (see Fig. 6).

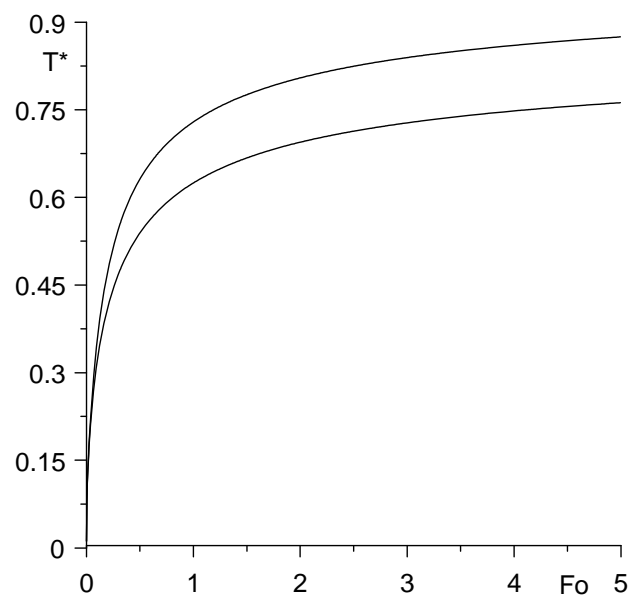

Fig. 6. Evolution of dimensionless temperature $T^{(0)^{*}} / A$ in the centre of heated zone for laser systems working in the continuous generation regime (Yevtushenko et al., 2009). 
By making assumption that at some moment $\tau$, temperature in the centre of the heated zone reaches the melting temperature of material, then:

$$
T^{(0)^{*}}(0,0, \tau)=T_{m} / A T_{0}
$$

where temperature $T^{(0)^{*}}(0,0, \tau)$ is derived from formula (37) or (38).

The right side of the Eq. (39) includes material characteristic features such as: thermal conductivity $K$ and melting temperature $T_{m}$, the laser beam characteristic parameters: heat flux intensity $q_{0}$, laser beam radius $a$ and effective absorption coefficient $A$. If all the values are known then from Eq. (39) the dimensionless boundary time point of melting beginning can be found. By choosing for a specific material the appropriate laser beam parameters, the melting start point can be calculated and damage of a surface as a result of intensive melting can be avoided.

In work (Rozniakowski, 2001), for sample of steel St45:

- $K=33.5 \mathrm{~W} /(\mathrm{mK}), k=15 \cdot 10^{-6} \mathrm{~m}^{2} / \mathrm{s}, \mathrm{T}_{m}=1535^{\circ} \mathrm{C}$,

- heated by the Nd:YAG laser beam in system KWANT 15 ( $a=0.64 \mathrm{~mm}$ radius, laser pulse duration $t_{s}=2 m s$ ),

- $\quad q_{0}=0.7 \cdot 10^{9} \mathrm{~W} / \mathrm{m}^{2}$, when effective absorption coefficient is $A \approx 50 \%$ (Rozniakowski, 2001),

the below calculation were conducted with use of Eqs. (13), (39) and numerical results on Fig. 6. From Eq. (13) the $T_{0}=0,133 \cdot 10^{5} \mathrm{~K}$ was determined. When the right side of Eq. (39) reaches the value $T_{m} /\left(A T_{0}\right)=0.2308$, then on Fig. 6 the dimensionless time $\tau=0.043$ for the uniform distribution and respectively $\tau=0.047$ for the normal distribution can be found. These dimensionless time values have respective real time values: $t=1.17 \mathrm{~ms}$ and $t=1.28 \mathrm{~ms}$.

\subsection{Determination of laser irradiation effective absorption coefficient method}

It should be underlined that maximum temperature value (19) on the body surface is achieved at the moment of laser switiching off $t=t_{s} \quad\left(\tau=\tau_{s}\right)$ (Fig. 3). In order to determine the monochromatic effective absorption coefficient $A$, the value of the retardation time $\Delta t(\Delta \tau)$ is needed to be known (its value increases quickly with the distance from laser irradiated surface of the body). Retardation time $\Delta t(\Delta \tau)$ can be determined from the condition of the maximum temperature reached inside the semi-infinite body in the point of $(r, z)(\rho, \zeta)$ at $t=t_{h}\left(\tau=\tau_{h}\right):$

$$
\left.\frac{\partial T^{*}(\rho, \zeta, \tau)}{\partial \tau}\right|_{\tau=\tau_{h}}=0, \rho \geq 0, \zeta \geq 0
$$

Differentiating equations (19)-(22) with respect to dimensionless time $\tau$ we obtain

$$
\frac{\partial T^{*}(\rho, \zeta, \tau)}{\partial \tau}=A\left[\frac{\partial T^{(0) *}(\rho, \zeta, \tau)}{\partial \tau} H(\tau)-\frac{\partial T^{(0) *}\left(\rho, \zeta, \tau-\tau_{s}\right)}{\partial \tau} H\left(\tau-\tau_{\mathrm{s}}\right)\right],
$$

where 


$$
\frac{\partial T^{(0) *}(\rho, \zeta, \tau)}{\partial \tau}=\frac{1}{\sqrt{\pi \tau}} \int_{0}^{\infty} \varphi(\xi) \xi e^{-\left(\frac{\zeta^{2}}{4 \tau}+\xi^{2} \tau\right)} J_{0}(\xi \rho) d \xi .
$$

Taking into account the form of function $\varphi(\xi)$ given by (21), the Eq. (42) can be written as:

$$
\frac{\partial T^{(0) *}(\rho, \zeta, \tau)}{\partial \tau}=\left[f M_{1}(\rho, \tau)+(1-f) M_{2}(\rho, \tau)\right] \frac{e^{-\frac{\zeta^{2}}{4 \tau}}}{2 \sqrt{\pi \tau}},
$$

where (Prudnikov et al., 1998)

$$
M_{1}(\rho, \tau)=\int_{0}^{\infty} \xi e^{-\xi^{2}\left(\frac{1}{4}+\tau\right)} J_{0}(\xi \rho) d \xi=\frac{2}{1+4 \tau} e^{-\frac{\rho^{2}}{1+4 \tau}},
$$

and

$$
M_{2}(\rho, \tau)=\int_{0}^{\infty}\left(1-\frac{\xi^{2}}{4}\right) \xi e^{-\xi^{2}\left(\frac{1}{4}+\tau\right)} J_{0}(\xi \rho) d \xi=\left[1-\frac{1}{1+4 \tau}\left(1-\frac{\rho^{2}}{1+4 \tau}\right)\right] M_{1}(\rho, \tau)
$$

Substituting functions $M_{i}(\rho, \tau), i=1,2$ (44), (45) into Eq. (43), we got:

$$
\frac{\partial T^{(0) *}(\rho, \zeta, \tau)}{\partial \tau}=\frac{B_{f}\left[\left(1+4 B_{f} \tau\right)\left(f+4 B_{f} \tau\right)+(1-f) B_{f} \rho^{2}\right]}{\left(1+4 B_{f} \tau\right)^{3} \sqrt{\pi \tau}} e^{\left[-\left(\frac{B_{f} \rho^{2}}{1+4 B_{f} \tau}+\frac{\zeta^{2}}{4 \tau}\right)\right]} .
$$

By substituting the partial derivative (46) into Eq. (41), from condition (40) the nonlinear functional equation with respect to the dimensionless retardation time $\Delta \tau$ is obtained:

$$
C_{1} C_{2}^{3} D_{f}(\rho)=e^{-\left(C_{3} \rho^{2}+C_{4} Z^{2}\right)}, \rho \geq 0, Z \geq 0 \text {, }
$$

where

$$
C_{1}=\sqrt{1-\frac{\tau_{\mathrm{s}}}{\tau_{h}}}, C_{2}=\frac{4 B_{f} \tau_{\mathrm{s}}}{1+4 B_{f} \tau_{h}}, C_{3}=\frac{4 B_{f} \tau_{\mathrm{s}}}{\left(1+4 B_{f} \Delta \tau\right)\left(1+4 B_{f} \tau_{h}\right)}, C_{4}=\frac{\tau_{\mathrm{s}}}{4 \tau_{h} \Delta \tau}
$$

and

$$
D_{f}(\rho)=\frac{(1-f) B_{f} \rho^{2}+\left(1+4 B_{f} \tau_{h}\right)\left(f+4 B_{f} \tau_{h}\right)}{(1-f) B_{f} \rho^{2}+\left(1+4 B_{f} \Delta \tau\right)\left(f+4 B_{f} \Delta \tau\right)}
$$

By applying logarithm on (47) we have the following results:

$$
C_{3} \rho^{2}+C_{4} \zeta^{2}=\ln \left[C_{1} \cdot C_{2}^{3} \cdot D_{f}(\rho)\right]^{-1} \quad \rho \geq 0, \zeta \geq 0 .
$$


Eq. (50) defines the isotherm of maximum temperature for a given values of $\tau_{s}$ and $\Delta \tau$ (see Fig. 7). For the case of normal distribution of irradiation intensity $\left(f=1, B_{f}=1\right)$, the $D_{f}(\rho)$ function (49) is independent on dimensionless radial variable $\rho$ and can be expressed by:

$$
D_{f} \equiv D_{1}=\frac{\left(1+4 \tau_{h}\right)^{2}}{(1+4 \Delta \tau)^{2}}=\frac{1}{C_{2}^{2}},
$$

where $C_{2}$ is given by (48). By using relation (51), the Eq. (50) can be rewritten in the form:

$$
C_{3} \rho^{2}+C_{4} \zeta^{2}=\ln \left[C_{1} C_{2}\right]^{-1}, \rho \geq 0, \zeta \geq 0 .
$$

and consequently as:

$$
\frac{\rho^{2}}{\alpha^{2}}+\frac{\zeta^{2}}{\beta^{2}}=1,|\rho| \leq \alpha, 0<\zeta \leq \beta,
$$

where

$$
\alpha^{2}=-\frac{\ln \left(C_{1} C_{2}\right)}{C_{3}}, \beta^{2}=-\frac{\ln \left(C_{1} C_{2}\right)}{C_{4}} .
$$

By analysing the Eqs. (53), (54) it can be found that the isotherm of maximum dimensionless temperature for the normal (gaussian) distribution of irradiation intensity has the form of half-ellipse (see Fig. 7) with the axes given by (53) $-r_{h}=\alpha a, z_{h}=\beta a$. In case of the toroidal (ring) distribution of the heat flux, the isotherm takes the form of curve with maximum shifted from axial axis. On symmetry axis $\rho=0$, the Eq. (52) has form:

$$
C_{4} \zeta^{2}=\ln \left[C_{1} C_{2}\right]^{-1}
$$

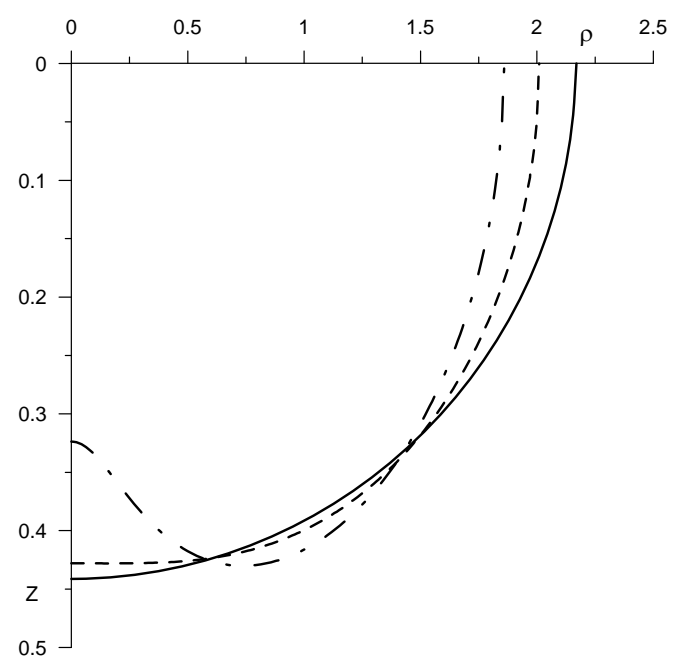

Fig. 7. Isotherms of maximum dimensionless temperature $T_{\max }^{*}$ at $\tau_{s}=0.672$ and $\Delta \tau=0.0054$ (Yevtushenko et al., 2009). 
By substituting in Eq. (55) the coefficients $C_{1}, C_{2}$ and $C_{4}$ respectively (50), the following is derived:

$$
\zeta_{h}=\left\{\frac{4 \Delta \tau\left(\tau_{s}+\Delta \tau\right)}{\tau_{s}} \ln \left[\sqrt{\frac{\Delta \tau}{\tau_{s}+\Delta \tau}}\left(\frac{1+4 \Delta \tau}{1+4\left(\tau_{s}+\Delta \tau\right)}\right)\right]^{-1}\right\}^{1 / 2} .
$$

From (56) equation, when retardation time $\Delta \tau$ and heating time $\tau_{s}$ are known, the maximum dimensionless layer depth $\zeta_{h}$ in irradiated material, where phase transitions occur, can be found. In the contrary case, when maximum dimensionless layer depth $\zeta_{h}$ in irradiated material is known (the temperature $T$ exceeded some critical value $T_{h}$ and phase transition took place), then from Eq. (56) the dimensionless retardation time $\Delta \tau$ can be found, too. From condition $T\left(r, z, t_{h}\right)=T_{h}$, where $T$ is given from solution (19)-(22), the following formula for determination of the effective absorption coefficient, is obtained:

$$
A=A^{*} \frac{T_{h}}{T_{0}}
$$

where

$$
A^{*}=\left\{\int_{0}^{\infty} \varphi(\xi)\left[\Phi\left(\xi, \zeta_{h}, \tau_{s}\right)-\Phi\left(\xi, \zeta_{h}, \Delta \tau\right)\right] J_{0}\left(\xi \rho_{h}\right) d \xi\right\}^{-1},
$$

and coordinates $\left(\rho_{h}, \zeta_{h}\right)$ fulfill the Eq. (53).

\subsection{Experimental results}

Some results of steel 45 type hardening by laser irradiation can be found in work (Rozniakowski, 2001). The application of determination of effective absorption coefficient method is also there included on the basis of effective reflection coefficient $R(A=1-R)$. Characteristic features of mentioned above material and laser system applied in the experiment are gathered in Table 1 . The steel 45 type sample had rounded shape of $20 \mathrm{~mm}$ diameter and $6 \mathrm{~mm}$ in thickness, the percentage of other elements had following distribution: C $-0.42 \div 0,50 \%$, Mn $-0.5 \div 0,8 \%$, Si- $0.17 \div 0.37 \%$, P- $0.040 \%$, S $-0.045 \%$. Laser system was working in free generation regime (Nd:YAG laser type, $\lambda=1.06 \mu \mathrm{m}, E_{i}=1.5 \mathrm{~J}$ and laser pulse time duration $t_{s}=2 \mathrm{~ms}$ ).

\begin{tabular}{|c|c|c|c|c|}
\hline parameters & $\begin{array}{c}K, \\
{\left[\mathrm{Wm}^{-1} \mathrm{~K}^{-1}\right]}\end{array}$ & $\begin{array}{c}k \times 10^{5}, \\
{\left[\mathrm{~m}^{2} \mathrm{~s}^{-1}\right]}\end{array}$ & $\begin{array}{c}T_{h}, \\
{[\mathrm{~K}]}\end{array}$ & $\begin{array}{c}z_{h}, \\
{[\mu \mathrm{m}]}\end{array}$ \\
\hline steel 45 & 33.50 & 1.50 & 1123 & 40 \\
\hline
\end{tabular}

Table 1. Characteristic features of steel 45 type.

Steel sample was heated locally by laser irradiation beam in 10 different points with changing irradiation intensity. Subsequently, the metalographic cross-section were done for 
the irradiated areas. By using the EPITYP-2 metalographic microscope and SEM TESLA BS300 the maximum hardened layer depth $z_{h}$ were measured (see Fig. 8).

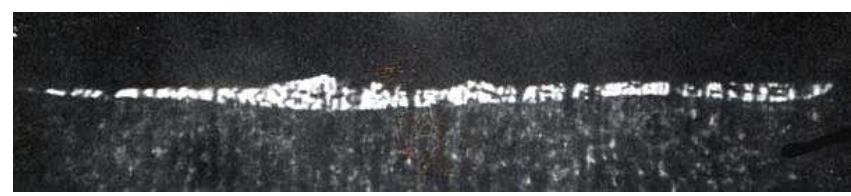

Fig. 8. Metalographic cross-section of laser irradiated steel sample.

The diameters of hardened depths were much larger than $z_{h}$, it was also observed the melting point starts when $q_{0}>8.5 \cdot 10^{8} \mathrm{~W} / \mathrm{m}^{2}$ where:

$$
q_{0}=\frac{E_{i}}{\pi a^{2} t_{s}} .
$$

Moreover, it was found that for $q_{0}=5.8 \cdot 10^{8} \mathrm{~W} / \mathrm{m}^{2}$, the hardened layer depth $z_{h}$ equals $40 \mu \mathrm{m}$. By using Eq. (59) the radius of irradiated area can be found as $a=0.64 \mathrm{~mm}$ and consequently the dimensionless irradiation time $\tau_{s}$. Hence from Eq. (56) at $\zeta_{h}=z_{h} / a$ the dimensionless retardation time $\Delta \tau$, was obtained (see Table 2). For such dimensionless coordinates values $\left(\rho_{h}=0, \zeta_{h}\right)$ and dimensionless time values $\left(\tau_{s}, \Delta \tau\right)$, from Eq. (58) the parameter $A^{*}=4.5$ was calculated, and from Eq.(57) the real effective absorption coefficient A was found, see comparison of the experimental and analytical values gathered in Table 2, (Rozniakowski, 2001).

\begin{tabular}{|c|c|c|c|c|c|c|}
\hline parameters & $\begin{array}{c}T_{0} \times 10^{-5}, \\
{\left[\mathrm{~K}^{-1}\right]}\end{array}$ & $\begin{array}{c}\zeta_{h}, \\
{[-]}\end{array}$ & $\begin{array}{c}\tau_{s}, \\
{[-]}\end{array}$ & $\begin{array}{c}\Delta \tau \times 10^{3} \\
{[-]}\end{array}$ & $\begin{array}{c}A \\
\text { experimental }\end{array}$ & $\begin{array}{c}A \\
\text { from Eq. (57) }\end{array}$ \\
\hline steel 45 & 0.111 & 0.062 & 0.073 & 0.33 & $30 \% \div 50 \%$ & $41.8 \%$ \\
\hline
\end{tabular}

Table 2. Comparison of the experimental and analytical values of the effective absorption coefficient $A$.

\section{Quasi-static thermal stresses caused by laser irradiation heating}

\subsection{Non-stationary temperature field}

By using variables and parameters (13), the axisymmetrical boundary value problem of transient heat conduction for semi-infinite body can be considered as:

$$
\begin{gathered}
\frac{\partial^{2} T^{*}}{\partial \rho^{2}}+\frac{1}{\rho} \frac{\partial T^{*}}{\partial \rho}+\frac{\partial^{2} T^{*}}{\partial \zeta^{2}}=\frac{\partial T^{*}}{\partial \tau}, \rho \geq 0, \zeta>0, \tau>0, \\
T^{*}(\rho, \zeta, 0)=0, \rho \geq 0, \zeta \geq 0,
\end{gathered}
$$




$$
\begin{gathered}
\frac{\partial T^{*}}{\partial \zeta}-B i T^{*}=-q^{*}(\rho) H(\tau), \rho \geq 0, \zeta=0, \tau>0, \\
T^{*}(\rho, \zeta, \tau) \rightarrow 0, \sqrt{\rho^{2}+\zeta^{2}} \rightarrow \infty, \tau>0,
\end{gathered}
$$

where from Eq. (18) for $f=1$ following is given

$$
q^{*}(\rho)=e^{-\rho^{2}}, \rho \geq 0
$$

By applying the Hankel integral transformation along the radial $\rho$ (Sneddon, 1972)

$$
\bar{T}^{*}(\xi, \zeta, \tau)=\int_{0}^{\infty} \rho T^{*}(\rho, \zeta, \tau) J_{0}(\xi \rho) d \rho,
$$

to the boundary heat conduction problem (60)-(63) then it is denoted

$$
\begin{gathered}
\frac{\partial^{2} \bar{T}^{*}}{\partial \zeta^{2}}-\xi^{2} \bar{T}^{*}=\frac{\partial \bar{T}^{*}}{\partial \tau}, \\
\bar{T}^{*}(\xi, \zeta, 0)=0, \\
\bar{T}^{*}(\xi, \infty, \tau)=0, \\
\frac{\partial \bar{T}^{*}}{\partial Z}=-\varphi(\xi)+B i \bar{T}^{*}, \zeta=0,
\end{gathered}
$$

where after consideration of Eq. (64)

$$
\varphi(\xi)=\int_{0}^{\infty} \rho e^{-\rho^{2}} J_{0}(\xi \rho) d \rho=\frac{1}{2} e^{-\frac{\xi^{2}}{4}} .
$$

When applying Fourier integral transformation with generalized trigonometric kernel along $\zeta$ variable to the boundary value problem (66)-(69) (Sneddon, 1972) then

$$
\tilde{\bar{T}}^{*}(\xi, \varsigma, \tau)=\sqrt{\frac{2}{\pi}} \int_{0}^{\infty} \bar{T}^{*}(\xi, \zeta, \tau) N(\zeta, \varsigma) d \zeta, N(\zeta, \varsigma)=\varsigma \cos (\varsigma \zeta)+B i \sin (\varsigma \zeta) .
$$

As a result of transformation the Cauchy problem is obtained in the form:

$$
\begin{gathered}
\frac{d \bar{T}^{*}}{d \tau}+\left(\varsigma^{2}+\xi^{2}\right) \tilde{\bar{T}}^{*}=\sqrt{\frac{2}{\pi}} \varsigma \varphi(\xi), \tau>0, \\
\tilde{\bar{T}}^{*}(\xi, \varsigma, 0)=0 .
\end{gathered}
$$

Solution of the ordinary differential equation (72) with initial condition (73) has form 


$$
\tilde{\bar{T}}^{*}(\xi, \varsigma, \tau)=\varphi(\xi) \tilde{\Phi}_{0}(\xi, \varsigma, \tau)
$$

where

$$
\tilde{\Phi}_{0}(\xi, \varsigma, \tau)=\sqrt{\frac{2}{\pi}} \frac{\varsigma}{\varsigma^{2}+\xi^{2}}\left[1-e^{-\left(\varsigma^{2}+\xi^{2}\right) \tau}\right] .
$$

By applying to the solutions (74), (75) below listed Fourier and Hankel inverted integral transformations (Sneddon, 1972):

$$
\begin{gathered}
\bar{T}^{*}(\xi, \zeta, \tau)=\sqrt{\frac{2}{\pi} \int_{0}^{\infty} \frac{N(\zeta, \varsigma)}{\varsigma^{2}+B i^{2}} \tilde{\bar{T}}^{*}(\xi, \varsigma, \tau) d \varsigma,} \\
T^{*}(\rho, \zeta, \tau)=\int_{0}^{\infty} \xi \bar{T}^{*}(\xi, \zeta, \tau) J_{0}(\xi \rho) d \xi,
\end{gathered}
$$

the following will be obtained

$$
T^{*}(\rho, \zeta, \tau)=\int_{0}^{\infty} \xi \varphi(\xi) \Phi(\xi, \zeta, \tau) J_{0}(\xi \rho) d \xi, \rho \geq 0, \zeta>0, \tau>0
$$

where

$$
\begin{aligned}
\Phi(\xi, \zeta, \tau)=\frac{1}{2}\left[\frac{e^{-\xi \zeta}}{B i+\xi} \operatorname{erfc}\left(\frac{\zeta}{2 \sqrt{\tau}}-\xi \sqrt{\tau}\right)\right. & \left.+\frac{e^{\xi \zeta}}{B i-\xi} \operatorname{erfc}\left(\frac{\zeta}{2 \sqrt{\tau}}+\xi \sqrt{\tau}\right)\right]- \\
& -\frac{B i e^{B i \zeta}}{B i^{2}-\xi^{2}} e^{\left(B i^{2}-\xi^{2}\right) \tau} \operatorname{erfc}\left(\frac{\zeta}{2 \sqrt{\tau}}+B i \sqrt{\tau}\right)
\end{aligned} .
$$

It should be underlined that at $\xi \rightarrow B i$, function $\Phi(\xi, \zeta, \tau)(79)$ equals

$$
\Phi(B i, \zeta, \tau)=\frac{1}{4 B i} e^{-B i \zeta} \operatorname{erfc}\left(\frac{\zeta}{2 \sqrt{\tau}}-B i \sqrt{\tau}\right) .
$$

In case, when convection cooling does not occur on the semi-infinite surface of the body ( $B i=0)$, function $\Phi(\xi, \zeta, \tau)$ (79) gets form (81). At $\tau \rightarrow \infty$ from solution (78), (79) the stationary temperature in the centre of heated zone $\rho=0, \zeta=0$, is obtained:

$$
T_{\max }^{*}(0,0, \infty)=\frac{\sqrt{\pi}}{2}-\frac{B i}{2} \int_{0}^{\infty} \frac{e^{-\xi^{\frac{2}{4}}}}{B i+\xi} d \xi .
$$

From solution (81) at $B i \rightarrow 0$ the maximum temperature value without convection heat exchange, can be found (89).

\subsection{Quasi-static thermal stresses}

Non-uniform temperature distribution (78), (79) initiates in the semi-infinite surface of the body a field of thermal stresses. Thermal displacement $u_{i}$ which occurs in the elastic body 
as the result of temperature field interaction, without mass forces, can be found from differential equations system in partial differentials (Nowacki, 1986; Timoshenko \& Goodier, 1970):

$$
u_{i, j j}+\frac{1}{1-2 v} u_{j, j i}=\frac{2(1+v)}{1-2 v} \alpha_{t} T_{, i} .
$$

By introducing thermoelastic potential $\psi(\rho, \zeta, \tau)$ with use of formulae (Nowacki, 1986):

$$
u_{r}=\frac{1}{a} \frac{\partial \psi}{\partial \rho}, \quad u_{z}=\frac{1}{a} \frac{\partial \psi}{\partial \zeta},
$$

then equations system (82) can be rewritten in the form of equivalent Poisson equation:

$$
\nabla^{2} \psi=\beta_{t} a^{2} T_{0} T^{*}
$$

where $\beta_{t}=\alpha_{t} \frac{1+v}{1-v}$, and dimensionless temperature $T^{*}(\rho, \zeta, \tau)$ is given by (78), (79).

In case, when thermoelastic potential $\psi(\rho, \zeta, \tau)$ is known, then respective thermal stresses can be derived from formulae (Nowacki, 1986):

$$
\sigma_{r r}^{\psi}=\frac{2 \mu}{a^{2}}\left(\frac{\partial^{2} \psi}{\partial \rho^{2}}-\nabla^{2} \psi\right), \quad \sigma_{\theta \theta}^{\psi}=\frac{2 \mu}{a^{2}}\left(\frac{1}{\rho} \frac{\partial \psi}{\partial \rho}-\nabla^{2} \psi\right), \quad \sigma_{z z}^{\psi}=\frac{2 \mu}{a^{2}}\left(\frac{\partial^{2} \psi}{\partial \zeta^{2}}-\nabla^{2} \psi\right), \quad \sigma_{r z}^{\psi}=\frac{2 \mu}{a^{2}} \frac{\partial^{2} \psi}{\partial \rho \partial \zeta} .
$$

Solution of the Poisson equation (84) obtained with the use of Hankel integral transformations of zero order and Laplace integral transformations, which fulfilled the boundary condtions:

$$
\begin{gathered}
\sigma_{z z}^{\psi}=0\left(\frac{\partial^{2} \psi}{\partial \zeta^{2}}-\nabla^{2} \psi=0\right), \zeta=0, \rho \geq 0, \tau \geq 0 \\
\sigma_{r z}^{\psi}=0\left(\frac{\partial^{2} \psi}{\partial \rho \partial \zeta}=0\right), \zeta=0, \rho \geq 0, \tau \geq 0 \\
u_{r}=0\left(\frac{\partial \psi}{\partial \rho}=0\right), \zeta \geq 0, \rho=0, \tau \geq 0 \\
u_{r} \rightarrow 0\left(\frac{\partial \psi}{\partial \rho} \rightarrow 0\right), u_{z} \rightarrow 0\left(\frac{\partial \psi}{\partial \zeta} \rightarrow 0\right), \sqrt{\rho^{2}+\zeta^{2}} \rightarrow \infty
\end{gathered}
$$

has form

$$
\psi(\rho, \zeta, \tau)=T_{0} \beta_{t} \int_{0}^{\infty} \xi \varphi(\xi) \Phi^{\psi}(\xi, \zeta, \tau) J_{0}(\xi \rho) d \xi
$$

where 


$$
\begin{gathered}
\Phi^{\psi(\xi, \zeta, \tau)}=\frac{1}{B i^{2}-\xi^{2}}\left\{\left(B i \tau+\frac{\zeta}{2}+\frac{B i}{B i^{2}-\xi^{2}}\right) \Phi^{+}(\xi, \zeta, \tau)-\right. \\
\left.-\left[\xi \tau+\frac{B i \zeta}{2 \xi}-\frac{1}{2 \xi}+\frac{B i^{2}}{\xi\left(B i^{2}-\xi^{2}\right)}\right] \Phi^{-}(\xi, \zeta, \tau)-\sqrt{\frac{\tau}{\pi}} e^{-\left(\xi^{2} \tau+\frac{\zeta^{2}}{4 \tau}\right)}\right\}- \\
-\frac{B i e^{B i \zeta}}{B i^{2}-\xi^{2}} e^{\left(B i^{2}-\xi^{2}\right) \tau} \operatorname{erfc}\left(\frac{\zeta}{2 \sqrt{\tau}}+B i \sqrt{\tau}\right), \\
\Phi^{ \pm}(\xi, \zeta, \tau)=\frac{1}{2}\left[e^{-\xi \zeta} \operatorname{erfc}\left(\frac{\zeta}{2 \sqrt{\tau}}-\xi \sqrt{\tau}\right) \pm \operatorname{erfc}\left(\frac{\zeta}{2 \sqrt{\tau}}+\xi \sqrt{\tau}\right)\right] .
\end{gathered}
$$

By substituting the potential $\psi(91)-(93)$ to formulae (86) the following is obtained:

$$
\sigma_{i j}^{\psi^{*}}(\rho, \zeta, \tau)=\int_{0}^{\infty} \varphi(\xi) S_{i j}^{\psi}(\xi, \rho, \zeta, \tau) d \xi-\delta_{i j} T^{*}(\rho, \zeta, \tau), \rho \geq 0, \zeta \geq 0, \tau \geq 0
$$

where

$$
\begin{gathered}
S_{r r}^{\psi}(\xi, \rho, \zeta, \tau)=\Phi^{\psi}(\xi, \zeta, \tau)\left[\frac{\xi^{2}}{\rho} J_{1}(\xi \rho)-\xi^{3} J_{0}(\xi \rho)\right], \\
S_{\theta \theta}^{\psi}(\xi, \rho, \zeta, \tau)=-\Phi^{\psi}(\xi, \zeta, \tau) \frac{\xi^{2}}{\rho} J_{1}(\xi \rho), \\
S_{z z}^{\psi}(\xi, \rho, \zeta, \tau)=\Phi^{\psi}(\xi, \zeta, \tau) \xi^{3} J_{0}(\xi \rho), \\
\Phi_{, \zeta}^{\psi}(\xi, \zeta, \tau)=\frac{1}{B i^{2}-\xi^{2}}\left\{\left(\xi^{2} \tau+\frac{B i \zeta}{2}+\frac{B i^{2}}{B i^{2}-\xi^{2}}\right) \Phi^{+}(\xi, \zeta, \tau)\right. \\
-\left(\xi B i \tau+\frac{B i}{2 \xi}-\frac{\xi \zeta}{2}+\frac{\xi B i}{B i^{2}-\xi^{2}}\right) \Phi^{-}(\xi, \zeta, \tau)-B i \sqrt{\frac{\tau}{\pi}} e-\left(\xi^{2} \tau+\frac{\zeta^{2}}{4 \tau}\right) \\
-\frac{B i^{2} e^{B i \zeta}}{B i^{2}-\xi^{2}} e^{\left(B i^{2}-\xi^{2}\right) \tau} \operatorname{erfc}\left(\frac{\zeta}{2 \sqrt{\tau}}+B i \sqrt{\tau}\right), \\
\sigma_{i j}^{\psi *}=\frac{\sigma_{i j}^{\psi}}{\sigma_{0}}, \sigma_{0}=2 \mu \beta_{t} T_{0},
\end{gathered}
$$

and where $T^{*}(\rho, \zeta, \tau)$ - dimensionless temperature (78), (79), $\delta_{r r}=\delta_{\theta \theta}=1, \delta_{z z}=\delta_{r z}=0$. 
It should be noticed that on semi-infinite surface of the body $\zeta=0$ the boundary condition (88) is not fulfilled, which means that $\sigma_{r z}^{\psi^{*}} \neq 0$. Thus, the additional problem of forces interacting on semi-infinite surface of the body, which have such distribution that stresses $\sigma_{i j}^{L *}=\sigma_{i j}^{L} / \sigma_{0}$ caused by them together with stresses field $\sigma_{i j}^{\psi^{*}}(94)-(100)$, will fulfill the boundary condition, should be investigated:

$$
\sigma_{z z}^{\psi^{*}}+\sigma_{z z}^{L *}=0, \sigma_{r z}^{\psi *}+\sigma_{r z}^{L *}=0, \zeta=0, \rho \geq 0, \tau \geq 0 .
$$

Stresses field caused by the forces axisymmetrically distributed on the elastic semi-infinite surface of the body are derived with use of the Love function $L(\rho, \zeta, \tau)$ from biharmonic equation (Abramowitz \& Stegun, 1979):

$$
\nabla^{4} L=0
$$

Stresses tensor components are related with the Love function $L(\rho, \zeta, \tau)$ by relation (Nowacki, 1986)

$$
\begin{aligned}
& \sigma_{r r}^{L}=\frac{1}{a^{3}} \frac{\partial}{\partial \zeta}\left(v \nabla^{2} L-\frac{\partial^{2} L}{\partial \rho^{2}}\right), \quad \sigma_{\theta \theta}^{L}=\frac{1}{a^{3}} \frac{\partial}{\partial \zeta}\left(v \nabla^{2} L-\frac{1}{\rho} \frac{\partial L}{\partial \rho}\right), \\
& \sigma_{z z}^{L}=\frac{1}{a^{3}} \frac{\partial}{\partial \zeta}\left[(2-v) \nabla^{2} L-\frac{\partial^{2} L}{\partial \zeta^{2}}\right], \quad \sigma_{r z}^{L}=\frac{1}{a^{3}} \frac{\partial}{\partial \rho}\left[(1-v) \nabla^{2} L-\frac{\partial^{2} L}{\partial \zeta^{2}}\right] .
\end{aligned}
$$

Solution of equation (102), which fulfills the boundary conditions (101), is declining in infinity and has form:

$$
L(\rho, \zeta, \tau)=\frac{2 v-1}{2 \mu} \sigma_{0} a^{3} \int_{0}^{\infty} \varphi(\xi) e^{-\xi \zeta} \cdot\left\{(2 v+2+\xi \zeta) \Phi^{\psi}(\xi, 0, \tau)+\left(\frac{2 v+1}{\xi}+\zeta\right) \Phi_{, \zeta}^{\psi}(\xi, 0, \tau)\right\} J_{0}(\xi \rho) d \xi
$$

Dimensionless stresses $\sigma_{i j}^{L *}$ pertaining to $L(\rho, \zeta, \tau)$ (104) can be found by using formulae (103):

$$
\sigma_{i j}^{L *}(\rho, \zeta, \tau)=\int_{0}^{\infty} \varphi(\xi) \xi^{2} S_{i j}^{L}(\xi, \rho, \zeta, \tau) d \xi, \rho \geq 0, \zeta \geq 0, \tau \geq 0
$$

where

$$
\begin{aligned}
& S_{r r}^{L}(\xi, \rho, \zeta, \tau)=\left[(\xi \zeta-1) \xi \Phi^{\psi}(\xi, 0, \tau)+(\xi \zeta-2) \Phi_{, \zeta}^{\psi}(\xi, 0, \tau)\right] J_{0}(\xi \rho)+ \\
&+\left[(1-2 v-\xi \zeta) \xi \Phi^{\psi}(\xi, 0, \tau)+(2-2 v-\xi \zeta) \Phi_{, \zeta}^{\psi}(\xi, 0, \tau)\right] \frac{J_{1}(\xi \rho)}{\xi \rho}, \\
& S_{\theta \theta}^{L}(\xi, \rho, \zeta, \tau)=-\left[2 v \xi \Phi^{\psi}(\xi, 0, \tau)+2 v \Phi_{, \zeta}^{\psi}(\xi, 0, \tau)\right] J_{0}(\xi \rho)+ \\
&+\left[(2 v-1+\xi \zeta) \xi \Phi^{\psi}(\xi, 0, \tau)+(2 v-2+\xi \zeta) \Phi_{, \zeta}^{\psi}(\xi, 0, \tau)\right] \frac{J_{1}(\xi \rho)}{\xi \rho}, \\
& S_{z z}^{L}(\xi, \rho, \zeta, \tau)=-\left[(1+\xi \zeta) \xi \Phi^{\psi}(\xi, 0, \tau)+\xi \zeta \Phi_{, \zeta}^{\psi}(\xi, 0, \tau)\right] J_{0}(\xi \rho),
\end{aligned}
$$




$$
S_{r z}^{L}(\xi, \rho, \zeta, \tau)=-\left[\xi \zeta \Phi^{\psi}(\xi, 0, \tau)-(1-\xi \zeta) \Phi_{, \zeta}^{\psi}(\xi, 0, \tau)\right] J_{1}(\xi \rho)
$$

Total field of dimensionless thermal stresses $\sigma_{i j}^{*}=\sigma_{i j} / \sigma_{0}$ is obtained with use of superposition of stresses field $\sigma_{i j}^{\psi^{*}}(94)-(100)$, related with thermoelastic potential $\psi$ and with stresses $\sigma_{i j}^{L *}(105)-(109)$ pertaining to the Love function $L$ (104), in the form:

$$
\sigma_{i j}^{*}(\rho, \zeta, \tau)=\int_{0}^{\infty} \varphi(\xi) S_{i j}(\xi, \rho, \zeta, \tau) d \xi-\delta_{i j} T^{*}(\rho, \zeta, \tau), \rho \geq 0, \zeta \geq 0, \tau \geq 0,
$$

where

$$
S_{i j}(\xi, \rho, \zeta, \tau)=S_{i j}^{\psi}(\xi, \rho, \zeta, \tau)+\xi^{2} S_{i j}^{L}(\xi, \rho, \zeta, \tau),
$$

and function $\varphi(\xi)$ is given by formula (70), and function $S_{i j}^{\psi}, S_{i j}^{L}$ - by formulae (95)-(99) and (106)-(109), respectively.

\subsection{Modelling of the laser thermocracking process}

The non-stationary temperature field (78), (79) and the thermal stresses (110), (111) can produce an initiation and propagation of cracks in the laser irradiated body, which can lead finally to the fracture. The description of this phenomenon is based on the brittle cracking Griffith and McClintock-Walsh theory (Griffith, 1924; McClintock \& Walsh, 1962). The normal stresses $\sigma_{n}$ acting in the point of the boundary of predicted crack, can be derived from (Yevtushenko et al., 1997):

$$
\sigma_{n}=\frac{1}{2}\left[\left(\sigma_{1}+\sigma_{3}\right)+\left(\sigma_{1}-\sigma_{3}\right) \cos \left(2 \theta^{*}\right)\right]
$$

where $\sigma_{1}$ and $\sigma_{3}$ denote $\mathrm{d}$ the maximal and minimal principal stresses, $\theta^{*}=0.5 \operatorname{arctg}\left(1 / f^{*}\right)$ denotes the angle of crack orientation measured anticlockwise from the direction of $\sigma_{3}$ and $f^{*}$ is the coefficient of surface friction.

If the normal stresses $\sigma_{n}$ lead to opening of the crack $\left(\sigma_{n}<0\right)$, then the brittle fracture of body can be determined by using the original or modified Griffith criteria (Griffith, 1924). The fracture process will be started at a given point of the body if

$$
\sigma_{1}=\sigma_{T} \wedge 3 \sigma_{1}+\sigma_{3}>0 \text {, where } \sigma_{T} \text { is the tensile strength of the material. }
$$

The propagation of the cracks occurs in the plane normal to the direction of principal stress $\sigma_{1}$. If at $\sigma_{n}<0$ in the given point of the body $3 \sigma_{1}+\sigma_{3}<0$, then to the initiation of fracture the modified Griffith condition (Griffith, 1924) is applied:

$$
-\frac{\left(\sigma_{1}-\sigma_{3}\right)^{2}}{\left(\sigma_{1}+\sigma_{3}\right)}=8 \sigma_{T}
$$

Crack propagation occurs in the plane inclined by angle $\theta$ to the direction of action of the maximum principal stress $\sigma_{1}$, where $\theta$ :

$$
\theta=0.5 \arccos \frac{\left(\sigma_{1}-\sigma_{3}\right)}{2\left(\sigma_{1}+\sigma_{3}\right)} .
$$


The fracture caused by compressive stresses in the point of crack margin $\left(\sigma_{n}>0\right)$ can be initiated there under the McClintock and Walsh condition (McClintock \& Walsh, 1962) when:

$$
\sigma_{3}-\sigma_{1} \frac{\sqrt{1+f^{* 2}}+f^{*}}{\sqrt{1+f^{* 2}}-f^{*}}=\sigma_{c} \text {, where } \sigma_{c} \text { is the compressive strength of material. }
$$

The fracture occurs in the direction of action of the major maximum stress $\sigma_{1}$.

\subsection{Numerical analysis and conclusions}

Dimensionless temperature $T^{*}(78),(79)$ on the irradiated surface of the body $\zeta=0$ for a different value of the $\rho$ variable is a transient process. Maximum of the dimensionless temperature $T^{*}$ is reached in the centre of the irradiated area $(\rho=0, \zeta=0)$ and approaches value 0.8862 , if $\tau \rightarrow \infty$. Value $\tau=10$ corresponds to the stationary state in the centre of irradiated area. If much closer to the centre of irradiation area $\rho=0$, then transient process is much shorter. For $\tau=1$ temperature in the point $\rho=0$ equals $75 \%$, in the point $\rho=1.5$ equals $12 \%$ of stationary value 0.8862 .

In the experiments, with use of the Nd:YAG laser system working in quasi-stationary generation regime, where emitted energy pulse has $E_{i}=35 \mathrm{~J}$ value, the radius of irradiation area was in range $0.1 \div 0.5 \mathrm{~mm}$ (see Fig. 9). Most of metals have thermal diffusivity coefficient around $k \approx 10^{-5} \mathrm{~m}^{2} / \mathrm{s}$, hence on the basis of achieved solutions the characteristic time of the transient process in the centre of the irradiated area, could be found as $0.1 \div 0.5 \mathrm{sec}$.

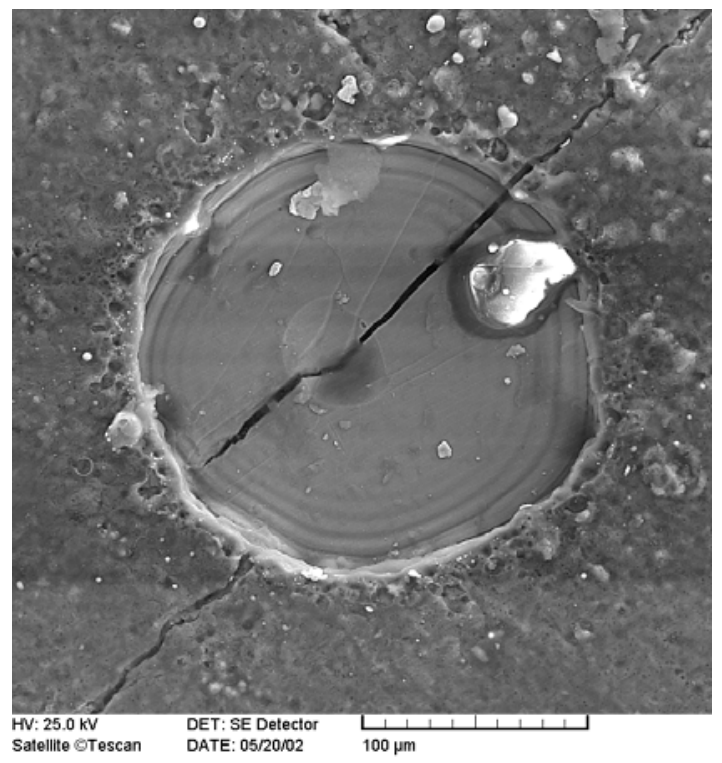

Fig. 9. SEM photograph of laser irradiated area of the Fe3\%Si steel. 


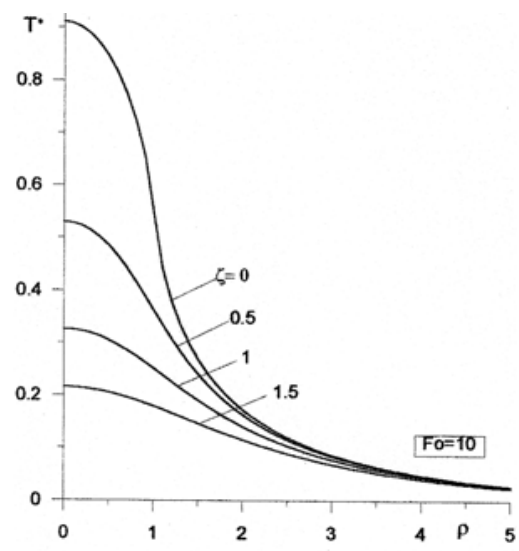

Fig. 10. Distribution of dimensionless stationary temperature $T^{*}$ along the dimensionless radial variable $\rho$ for $B i=0.01, \tau=10$ and for different values of dimensionless variable $\zeta$ (Rozniakowski et al., 2003).

Distribution of dimensionless stationary temperature along dimensionless variable $\zeta$ for different values of dimensionless radial variable $\rho$ is shown on Fig. 10. It can be noticed that the maximum temperatures and their higher gradients are achieved on the irradiated surface of the body in the centre of heated zone. Temperature increases quickly with the distance from the irradiated area and for $\zeta \geq 5 \quad(z \geq 5 a)$ reaches the starting value. Isotherms of dimensionless temperature $T^{*}$ are presented on Fig. 11. It can be noticed the isotherm surfaces of temperature are the rotation surfaces along $\rho$ axis. The highest temperature values and its highest gradients are concentrated close to the centre of laser irradiated area.

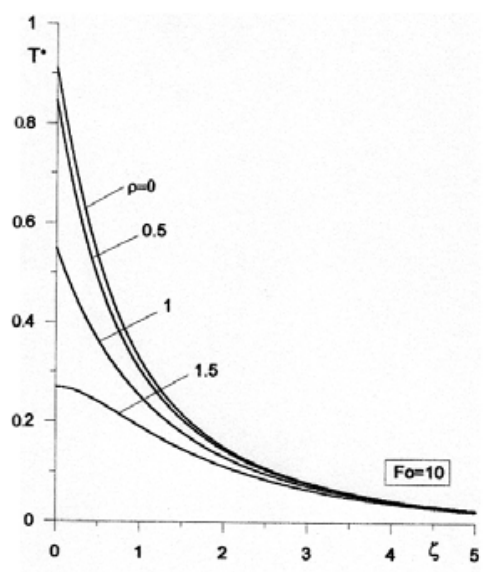

Fig. 11. Distribution of dimensionless temperature $T^{*}$ along dimensionless $\zeta$ variable for $B i=0.01, \tau=10$ and for different dimensionless values of $\zeta$ variable (Rozniakowski et al., 2003). 


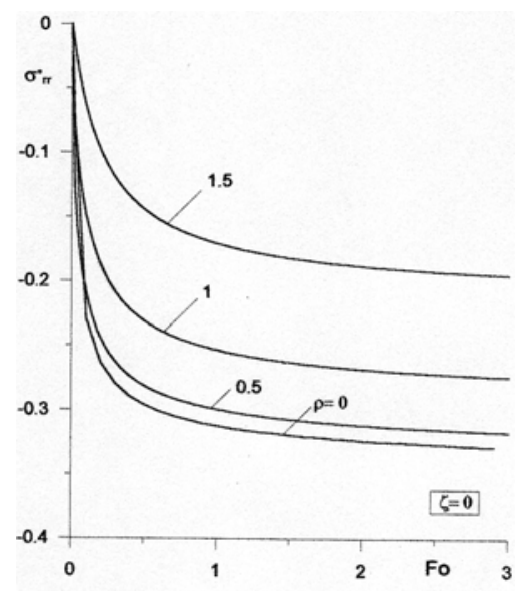

Fig. 12. Evolution of dimensionless radial stresses $\sigma_{r r}^{*}$ on the irradiated surface of the body $\zeta=0$ for $B i=0.01$ and different values of dimensionless radial variable $\rho$ (Rozniakowski et al., 2003).

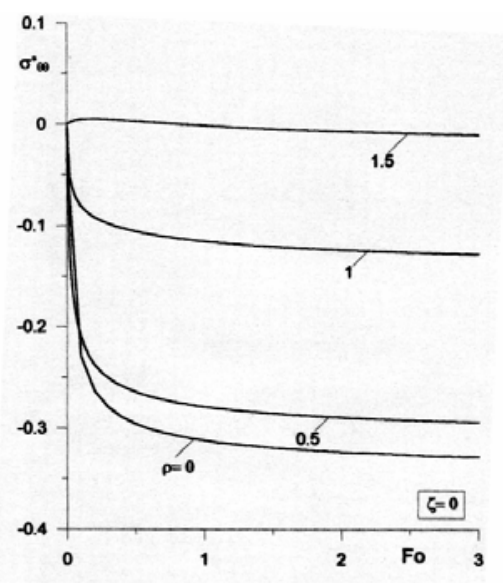

Fig. 13. Evolution of dimensionless peripheral stresses $\sigma_{\theta \theta}^{*}$ on the irradiated surface of the body $\zeta=0$ for $B i=0.01$ and for different values of dimensionless radial variable $\rho$ (Rozniakowski et al., 2003).

Evolution of dimensionless stresses with time $\sigma_{r r}^{*}$ and $\sigma_{\theta \theta}^{*}$ on the irradiated surface of the body $\zeta=0$ is shown on Figs. 12, 13. During irradiation process both components of stresses tensor are compressive and decrease with the distance from the centre of heated area. On the contrary, inside the body in the distance equal the radius of heated area $\zeta=1$, the normal stresses $\sigma_{z z}^{*}$ are stretching (see Fig. 14). With the beginning of laser irradiation process, these stresses increase quickly to the maximum value, and afterward decrease with 
time and reach the stationary value. The highest value of these stresses is achieved on symmetry axis $\rho=0$.

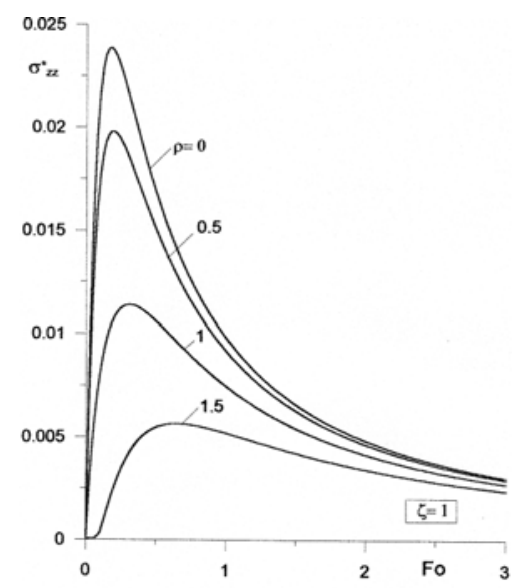

Fig. 14. Evolution of the dimensionless normal stresses $\sigma_{z z}^{*}$ on the plane $\zeta=1$ inside the irradiated body for $B i=0.01$ and for different values of dimensionless radial variable $\rho$ (Rozniakowski et al., 2003).

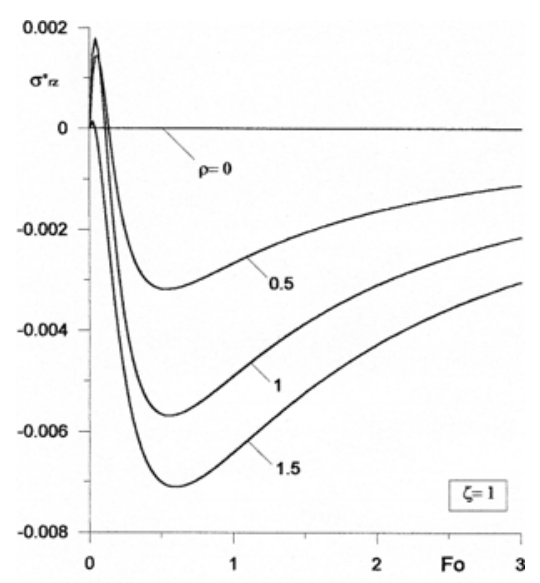

Fig. 15. Evolution of the dimensionless shear stresses $\sigma_{r z}^{*}$ on the plane $\zeta=1$ inside the irradiated body for $B i=0.01$ and for different values of dimensionless radial variable $\rho$ (Rozniakowski et al., 2003).

On the contrary to the normal stresses $\sigma_{z z}^{*}$, the shear stresses $\sigma_{r z}^{*}$ change their sign during laser irradiation process (see Fig. 15). In the very short time, after switching laser system on, the shear stresses are positive and afterward is changing to some negative value. With heating time the absolute values of stresses $\sigma_{r r}^{*}$ and $\sigma_{\theta \theta}^{*}$ increase, and stresses $\sigma_{z z}^{*}$ i $\sigma_{r z}^{*}$ 
values decrease. It should be underlined that accuracy of temperature and thermal stresses determination depends strongly on accuracy of heat exchange coefficient $h$ determination. The relation used in present calculations $h \approx 0.02 \mathrm{~K} / a$, under condition that convection heat exchange decreases the maximum temperature of the body not more than $10 \%$, was introduced in work (Rykalin et al., 1967).

\begin{tabular}{|c|c|c|c|}
\hline Parameters & Granite rock & Quart rock & Gabbro rock \\
\hline Uniaxial tensile strength, $T^{*}(\rho, \zeta, 0)=0[\mathrm{MPa}]$ & 9.0 & 13.5 & 16.0 \\
\hline Uniaxial compressive strength, $\rho \geq 0, \zeta \geq 0,[\mathrm{MPa}]$ & 205 & 190 & 162 \\
\hline Shear module, $\frac{\partial T^{*}}{\partial \zeta}=-q^{*}(\rho) I(\tau),[\mathrm{GPa}]$ & 28 & 36 & 34 \\
\hline Poisson coefficient $v$ & 0.23 & 0.16 & 0.24 \\
\hline Thermal conduction coefficient, $K[\mathrm{~W} / \mathrm{mK}]$ & 4.07 & 4.21 & 3.67 \\
\hline Thermal diffusivity coefficient $k \times 10^{-6}\left[\mathrm{~m}^{2} / \mathrm{s}\right]$ & 0.505 & 2.467 & 0.458 \\
\hline Linear thermal expansion coefficient $\alpha_{t} \times 10^{-6}[\mathrm{~K}-1]$ & 7.7 & 24.2 & 4.7 \\
\hline$T_{0} \times 10^{4}[\mathrm{~K}]$ & 0.246 & 0.237 & 0.272 \\
\hline$\sigma_{0}[\mathrm{GPa}]$ & 1.69 & 5.70 & 1.42 \\
\hline$\left(\sigma_{T} / \sigma_{0}\right) \times 10^{-3}$ & 5.319 & 2.367 & 11.280 \\
\hline
\end{tabular}

Table 3. Mechanical and thermo-physical features of granite, quart and gabbro taken from work (Yevtushenko et al., 1997).

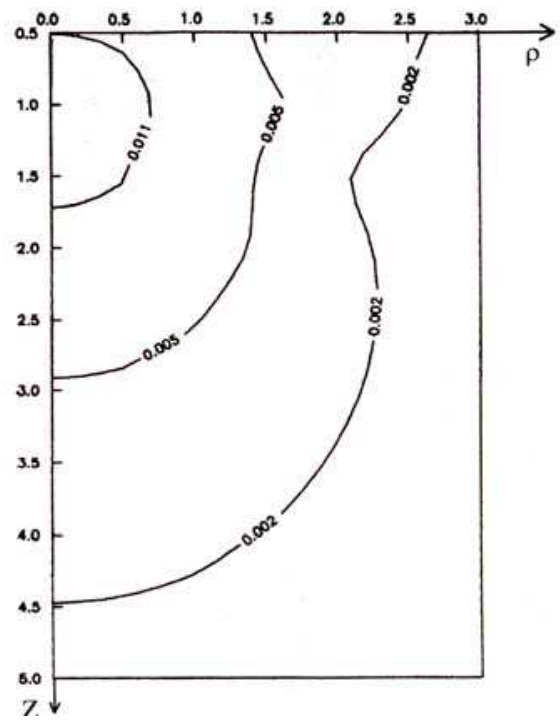

Fig. 16. Isolines of dimensionless major stresses $\sigma_{1}^{*}=\sigma_{T} / \sigma_{0}$ for features of materials from Table 3 (Yevtushenko et al., 2009). 
The maximum $\sigma_{1}^{*}=\sigma_{1} / \sigma_{0}$ and minimum $\sigma_{3}^{*}=\sigma_{3} / \sigma_{0}$ dimensionless major stresses are changing with the distance from irradiated surface of the body for different dimensionless time values $\tau$. The major stresses $\sigma_{1}^{*}$ are stretching for $\zeta>0$ and reach the maximum value close to the surface of semi-infinite half-space $\zeta \approx 0.8$ at the moment $\tau=0.1$. Other major stresses $\sigma_{3}^{*}$ are compressive during heating process and reach maximum value on the irradiated surface. By knowing distribution of major stresses $\sigma_{1}^{*}$ and $\sigma_{3}^{*}$, with use of criterial equations (113)-(116), the initiation and cracks propagation on the surface and inside the irradiated body, can be predicted. Substituting major stresses $\sigma_{1}^{*}$ and $\sigma_{3}^{*}$, calculated for $B i=0.01, \tau=0.1$, to the criterial equations (113)-(116) it was found that space below the heated surface of the body can be divided into three specific areas, in which each one of the criterial equations is fulfilled. In area $0 \leq \zeta \leq 0.4$ situated directly below heated surface of the body, the McClintock-Walsh equation (116) for the cracking caused by the compressive stresses, is fulfilled. In other area, where cracking is caused by shear stresses, the modified McClintock-Walsh equations (114), (115) are applied to their prediction. The maximum thickness of this area do not exceeded $0.5 a$ value. The area of stretching stresses is placed below the area in which compressive stresses are present. The Griffith criterion (113) is there applied.

On purpose of the numerical analysis three kinds of rocks were chosen: granite, quart, gabbro. The mechanical and thermo-physical features of these rocks material were taken from work (Yevtushenko et al., 1997) and gathered in Table 3. In Table 3, the constant values of $T_{0}(13)$ and $\sigma_{0}(100)$ were calculated for $q_{0}=10^{8} \mathrm{~W} / \mathrm{m}^{2}$ and $a=0.1 \mathrm{~mm}$. For these type materials the compressive strength $\sigma_{c}$ is much higher than the stretching strength $\sigma_{T}$. Hence, cracking process of such materials can be present in area where (113) criterion is applied and maximum major stresses $\sigma_{1}$ are equal to the stretching strength $\sigma_{T}$ :

$$
\sigma_{1}^{*}=\sigma_{T} / \sigma_{0}
$$

Set of points, in area where Griffith criterion (113) is fulfilled, is given in dimensionless form by (117) and form the isolines on the $\rho \zeta$ plane. Isolines of 0.002 value (quart), 0.005 value (granite) and 0.011 (gabbro) are shown on Fig. 16.

\section{Axi-symmetrical transient boundary-value problem of heat conduction and quasi-static thermoelasticity for pulsed laser heating of the semi-infinite surface of the body}

\subsection{Problem statement}

The following axi-symmetrical boundary-value problem of heat conduction is under consideration:

$$
\begin{gathered}
\frac{\partial^{2} T^{*}}{\partial \rho^{2}}+\frac{1}{\rho} \frac{\partial T^{*}}{\partial \rho}+\frac{\partial^{2} T^{*}}{\partial \zeta^{2}}=\frac{\partial T^{*}}{\partial \tau}, \rho \geq 0, \zeta>0, \tau>0, \\
T^{*}(\rho, \zeta, 0)=0, \rho \geq 0, \zeta \geq 0 \\
\frac{\partial T^{*}}{\partial \zeta}=-q^{*}(\rho) I(\tau),, \rho \geq 0, \zeta=0, \tau>0
\end{gathered}
$$




$$
T^{*}(\rho, \zeta, \tau) \rightarrow 0, \sqrt{\rho^{2}+\zeta^{2}} \rightarrow \infty, \tau>0,
$$

where dimensionless parameters were definied by formulae (13). Likewise in 3.1 sub-chapter it assumed that laser spatial irradiation intensity is normal (Hector \& Hetnarski, 1996):

$$
q^{*}(\rho)=e^{-\rho^{2}}, \rho \geq 0,
$$

and function $I(\tau)$ describing the change of laser irradiation intensity with time has form

$$
I(\tau)=I^{*} \exp \left[-\gamma\left(\tau^{\delta}-\tau_{r}^{\delta}\right)\right]\left(\frac{\tau}{\tau_{r}}\right)^{\beta}, \tau>0 .
$$

Because of the fact that accurate solution of boundary-value problem of heat conduction (118)-(121) for $I(\tau)$ (123) was not found the below method of approximation was applied.

\subsection{Laser pulse of rectangular shape}

Solution of the axi-symmetrical boundary-value problem of heat conduction (118)-(121) for normal spatial distribution of heat irradiation intensity (122) and constant with time

$$
I(\tau)=H(\tau), \tau \geq 0,
$$

has form (Carslaw \& Jaeger, 1959):

$$
T^{(0)^{*}}(\rho, \zeta, \tau)=\int_{0}^{\infty} \varphi(\xi) \Phi(\xi, \zeta, \tau) J_{0}(\xi \rho) d \xi, \rho \geq 0, \zeta \geq 0, \tau \geq 0
$$

where

$$
\varphi(\xi)=\int_{0}^{\infty} \rho q(\rho) J_{0}(\xi \rho) d \rho=\frac{1}{2} e^{-\frac{\xi^{2}}{4}}, \xi \geq 0,
$$

and function $\Phi(\xi, \zeta, \tau)(22)$.

Dimensionless quasi-static thermal stresses caused in the sem-infinite half-space by the nonstationary temperature field (125), which were achieved with use of the temperature potential methods and Love function (like in 3.2 sub-chapter) have form:

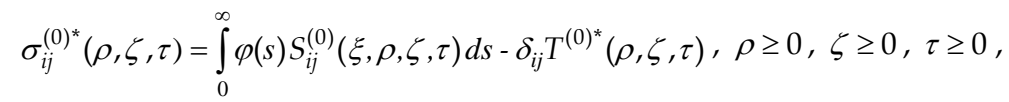

where

$$
\begin{aligned}
S_{r r}^{(0)}(\xi, \rho, \zeta, \tau) & =\Phi^{\psi}(\xi, \zeta, \tau)\left[\frac{\xi}{\rho} J_{1}(\xi \rho)-\xi^{2} J_{0}(\xi \rho)\right]- \\
-\xi^{2} e^{-\xi \zeta}\left\{-\left[(1-\xi \zeta) \Phi^{\psi}(\xi, 0, \tau)+(2-\xi \zeta) \Phi_{, \zeta}^{\psi}(\xi, 0, \tau)\right] J_{0}(\xi \rho)-\right. & \\
& \left.-\left[(2 v-1+\xi \zeta) \Phi^{\psi}(\xi, 0, \tau)+(2 v-2+\xi \zeta) \Phi_{, \zeta}^{\psi}(\xi, 0, \tau)\right] \frac{J_{1}(\xi \rho)}{(\xi \rho)}\right\},
\end{aligned}
$$




$$
\begin{gathered}
S_{\theta \theta}^{(0)}(\xi, \rho, \zeta, \tau)= \\
=-\Phi^{\psi}(\xi, \zeta, \tau) \xi \frac{J_{1}(\xi \rho)}{\rho}-\xi^{2} e^{-\xi \zeta}\left\{-2 v\left[\Phi^{\psi}(\xi, 0, \tau)+\Phi_{, \zeta}^{\psi}(\xi, 0, \tau)\right] J_{0}(\xi \rho)+\right. \\
\left.+\left[(2 v-1+\xi \zeta) \Phi^{\psi}(\xi, 0, \tau)+(2 v-2+\xi \zeta) \Phi_{, \zeta}^{\psi}(\xi, 0, \tau)\right] \frac{J_{1}(\xi \rho)}{(\xi \rho)}\right\}, \\
S_{z z}^{(0)}(\xi, \rho, \zeta, \tau)=\left\{\Phi^{\psi}(\xi, \zeta, \tau)-e^{-\xi \zeta}\left[(1+\xi \zeta) \Phi^{\psi}(\xi, 0, \tau)+\xi \zeta \Phi_{, \zeta}^{\psi}(\xi, 0, \tau)\right]\right\} \xi^{2} J_{1}(\xi \rho), \\
S_{r z}^{(0)}(\xi, \rho, \zeta, \tau)=\left\{-\Phi_{, \zeta}^{\psi}(\xi, \zeta, \tau)-e^{-\xi \zeta}\left[\xi \zeta \Phi^{\psi}(\xi, 0, \tau)-\right.\right. \\
\left.\left.-(1-\xi \zeta) \Phi_{, \zeta}^{\psi}(\xi, 0, \tau)\right]\right\} \xi^{2} J_{1}(\xi \rho) \\
\Phi^{\psi}(\xi, \zeta, \tau)=\left(\tau-\frac{1}{2 \xi^{2}}\right) \Phi^{-}(\xi, \zeta, \tau)-\frac{\zeta}{2 \xi} \Phi^{+}(\xi, \zeta, \tau)+\frac{1}{\xi} \sqrt{\frac{\tau}{\pi} e^{2}}\left[-\left(\xi^{2} \tau+\frac{\zeta^{2}}{4 \tau}\right)\right] \\
\Phi_{, \zeta}^{\psi}(\xi, \zeta, \tau)=\frac{\zeta}{2 \xi} \Phi^{-}(\xi, \zeta, \tau)-\tau \Phi^{+}(\xi, \zeta, \tau),
\end{gathered}
$$

and functions $T^{(0)^{*}}, \Phi^{ \pm}(\xi, \zeta, \tau)$ and factors $\delta_{i j}$ are defined in 3.2 sub-chapter. From solution (127)-(133) on the semi-infinite surface of the body $\zeta=0$ is received as follows: $\sigma_{z z}^{(0) *}(\rho, 0, \tau)=\sigma_{r z}^{(0) *}(\rho, 0, \tau)=0$. Solution for the rectangular-shape laser pulse:

$$
I(\tau)=H\left(\tau_{s}-\tau\right), \tau \geq 0,
$$

can be written in the form

$$
\begin{aligned}
& T^{*}(\rho, \zeta, \tau)=T^{(0) *}(\rho, \zeta, \tau) H(\tau)-T^{(0) *}\left(\rho, \zeta, \tau-\tau_{s}\right) H\left(\tau-\tau_{s}\right), \rho \geq 0, \zeta \geq 0, \tau \geq 0, \\
& \sigma_{i j}^{*}(\rho, \zeta, \tau)=\sigma_{i j}^{(0) *}(\rho, \zeta, \tau) H(\tau)-\sigma_{i j}^{(0) *}\left(\rho, \zeta, \tau-\tau_{s}\right) H\left(\tau-\tau_{s}\right), \rho \geq 0, \zeta \geq 0, \tau \geq 0,
\end{aligned}
$$

where dimensionless temperature $T^{(0) *}$ is determined from formulae (125), (126) and dimensionless thermal stresses $\sigma_{i j}^{(0) *}$ - by using Eqs. (127)-(133).

\subsection{Laser pulse of triangular shape}

Solution of the axi-symmetrical boundary-value problem of heat conduction (118)-(121) for normal spatial distribution of heat irradiation intensity (122) and linearly changing with time

$$
I(\tau)=\tau, \tau \geq 0
$$

has form

$$
T^{(1)^{*}}(\rho, \zeta, \tau)=\int_{0}^{\infty} \varphi(\xi) \Phi(\xi, \zeta, \tau) J_{0}(\xi \rho) d \xi, \rho \geq 0, \zeta \geq 0, \tau \geq 0
$$


where function $\varphi(\xi)$ is defined by Eq. (70), and $\Phi(\xi, \zeta, \tau)=\Phi^{\psi}(\xi, \zeta, \tau)$ (132). Dimensionless quasi-static thermal stresses generated in the semi-infinite surface of the body by the temperature field equal:

$$
\sigma_{i j}^{(1)^{*}}(\rho, \zeta, \tau)=\int_{0}^{\infty} \varphi(s) S_{i j}^{(1)}(\xi, \rho, \zeta, \tau) d s-\delta_{i j} T^{(1)^{*}}(\rho, \zeta, \tau), \rho \geq 0, \zeta \geq 0, \tau \geq 0,
$$

where functions $S_{i j}^{(1)}(\xi, \rho, \zeta, \tau)$ in solution (139) are derived from Eqs. (128)-(131) at:

$$
\begin{gathered}
\Phi^{\psi}(\xi, \zeta, \tau)=\frac{\zeta}{2 \xi}\left(\tau-\frac{3}{4 \xi^{2}}\right) \Phi^{+}(\xi, \zeta, \tau)-\left(\frac{\tau^{2}}{2}-\frac{\tau}{2 \xi^{2}}+\frac{\zeta}{8 \xi^{2}}+\frac{3}{8 \xi^{4}}\right) \times \\
\times \Phi^{-}(\xi, \zeta, \tau)-\left(\frac{\tau}{2}-\frac{3}{4 \xi^{2}}\right) \frac{1}{\xi} \sqrt{\frac{\tau}{\pi}} e^{\left[-\left(\xi^{2} \tau+\frac{\zeta^{2}}{4 \tau}\right)\right]}, \\
\Phi_{, \zeta}^{\psi}(\xi, \zeta, \tau)=\left(\frac{\tau^{2}}{2}+\frac{\zeta^{2}}{8 \xi^{2}}\right) \Phi^{+}(\xi, \zeta, \tau)+\frac{\zeta}{2 \xi}\left(\frac{1}{4 \xi^{2}}-\tau\right) \Phi^{-}(\xi, \zeta, \tau)- \\
-\frac{\zeta}{4 \xi^{2}} \sqrt{\frac{\tau}{\pi}} e^{\left[-\left(\xi^{2} \tau+\frac{\zeta^{2}}{4 \tau}\right)\right]} .
\end{gathered}
$$

Dimensionless temperature and respective dimensionless thermal stresses generated in the semi-infinite surface of the body by triangle-shape laser pulse can be found as the result of solutions superposition: for the constant (125), (127) and linear (138), (139) laser pulse shape of irradiation intensity:

$$
\begin{aligned}
& T^{*}(\rho, \zeta, \tau)= \frac{2}{\tau_{r}}\left[T^{(1) *}(\rho, \zeta, \tau)-T^{(1) *}\left(\rho, \zeta, \tau-\tau_{r}\right)\right]- \\
&-\frac{2}{\left(\tau_{s}-\tau_{r}\right)}\left[T^{(1) *}\left(\rho, \zeta, \tau-\tau_{r}\right)-T^{(1) *}\left(\rho, \zeta, \tau-\tau_{s}\right)\right], \\
& \sigma_{i j}^{*}(\rho, \zeta, \tau)=\frac{2}{\tau_{r}}\left[\sigma_{i j}{ }^{(1) *}(\rho, \zeta, \tau)-\sigma_{i j}{ }^{(1) *}\left(\rho, \zeta, \tau-\tau_{r}\right)\right]- \\
& \quad-\frac{2}{\left(\tau_{s}-\tau_{r}\right)} \sigma_{i j}^{(1) *}\left(\rho, \zeta, \tau-\tau_{r}\right)-\sigma_{i j}{ }^{(1) *}\left(\rho, \zeta, \tau-\tau_{s}\right) .
\end{aligned}
$$

\subsection{Laser pulse of any shape}

In this sub-chapter the laser pulse of any shape is under consideration. Solution of the axisymmetrical boundary-value problem of heat conduction (118)-(121) and respective thermoelasticity problem for semi-infinite surface of the body at laser pulse of any shape is found by the approximation method with the use of finite functions.

\section{Approximation by piecewise constant functions}

Closed interval $\langle 0, \tau\rangle$ will be divided in uniform net of points $\tau_{k}=k \delta \tau, k=0,1, \ldots, n$, gdzie $\delta \tau=\tau / n$. Set the following piecewise constant function in the form: 


$$
\varphi_{k}(\tau)=\left\{\begin{array}{l}
1, \tau \in<\tau_{k-1}, \tau_{k}>, \\
0, \tau \notin<\tau_{k-1}, \tau_{k}>, k=1,2, \ldots, n .
\end{array}\right.
$$

Function $I(\tau)$ is approximated by the function $\varphi_{k}(\tau)(144)$ in the form

$$
I(\tau) \approx \sum_{k=1}^{n} I\left(\bar{\tau}_{k}\right) \varphi_{k}(\tau), \bar{\tau}_{k}=\frac{\tau_{k-1}+\tau_{k}}{2}, \tau \geq 0
$$

The absolute accuracy of approximation given in (145) is around $O(\delta \tau)$. Hence, the solution of non-stationary boundary-value problem of heat conduction (118)-(121) with heat flux intensity of any laser pulse shape $I(\tau)$ can be written:

$$
T^{*}(\rho, \zeta, \tau)=\sum_{k=1}^{n} I\left(\bar{\tau}_{k}\right) T_{k}^{(0)^{*}}(\rho, \zeta, \tau), \rho \geq 0, \zeta \geq 0, \tau \geq 0,
$$

where

$$
T_{k}^{(0)^{*}}(\rho, \zeta, \tau)=T^{(0)^{*}}\left(\rho, \zeta, \tau-\tau_{k-1}\right)-T^{(0)^{*}}\left(\rho, \zeta, \tau-\tau_{k}\right),
$$

and dimensionless temperature $T^{(0)^{*}}$ is derived according to Eqs. (125), (126). Field of dimensionless thermal stresses caused in semi-infinite surface of the body by the temperature field (146), (147) is found in analogous way:

$$
\begin{gathered}
\sigma_{i j}{ }^{*}(\rho, \zeta, \tau)=\sum_{k=1}^{n} I\left(\bar{\tau}_{k}\right) \sigma_{i j, k}^{(0)^{*}}(\rho, \zeta, \tau), \rho \geq 0, \zeta \geq 0, \tau \geq 0, \\
\sigma_{i j, k}^{(0)^{*}}(\rho, \zeta, \tau)=\sigma_{i j}{ }^{(0)^{*}}\left(\rho, \zeta, \tau-\tau_{k-1}\right)-\sigma_{i j}{ }^{(0)^{*}}\left(\rho, \zeta, \tau-\tau_{k}\right),
\end{gathered}
$$

and dimenionless stresses $\sigma_{i j}^{(0)^{*}}$ are derived from Eqs. (127)-(133).

\section{Approximation by piecewise linear functions}

It is assumed that for the same time interval $\langle 0, \tau\rangle$ the identical uniform net of points as above is used. Set the following piecewise linear function in the form:

$$
\begin{aligned}
& \varphi_{0}(\tau)=\left\{\begin{array}{l}
\frac{\left(\tau_{1}-\tau\right)}{\delta \tau}, \tau \in<\tau_{0}, \tau_{1}>, \\
0, \tau \notin<\tau_{0}, \tau_{1}>,
\end{array} \quad \varphi_{k}(\tau)=\left\{\begin{array}{l}
\frac{\left(\tau-\tau_{k-1}\right)}{\delta \tau}, \tau \in<\tau_{k-1}, \tau_{k}>, \\
\frac{\left(\tau_{k+1}-\tau\right)}{\delta \tau}, \tau \in<\tau_{k}, \tau_{k+1}>, \\
0, \tau \notin<\tau_{k-1}, \tau_{k+1}>, k=1,2, \ldots, n-1
\end{array}\right.\right. \\
& \varphi_{n}(\tau)=\left\{\begin{array}{l}
\frac{\left(\tau-\tau_{n-1}\right)}{\delta \tau}, \tau \in<\tau_{n-1}, \tau_{n}>, \\
0, \tau \notin<\tau_{n-1}, \tau_{n}>.
\end{array}\right.
\end{aligned}
$$

Thus the approximation of $I(\tau)$ is done by the following subtotal 


$$
I(\tau) \approx \sum_{k=0}^{n} I\left(\tau_{k}\right) \varphi_{k}(\tau), \quad \tau>0 .
$$

Absolute approximation error (151) has order of $O\left(\delta \tau^{2}\right)$ (Marchuk \& Agoshkov, 1981). Hence the final solution will have form:

$$
T^{*}(\rho, \zeta, \tau)=\frac{1}{\delta \tau} \sum_{k=0}^{n} I\left(\tau_{k}\right) T_{k}^{(1)^{*}}(\rho, \zeta, \tau), \rho \geq 0, \zeta \geq 0, \tau \geq 0
$$

where

$$
\begin{gathered}
T_{0}^{(1)^{*}}(\rho, \zeta, \tau)=\tau_{1} T^{(0)^{*}}(\rho, \zeta, \tau)-T^{(1)^{*}}(\rho, \zeta, \tau)+T^{(1)^{*}}\left(\rho, \zeta, \tau-\tau_{1}\right), \\
T_{k}^{(1)^{*}}(\rho, \zeta, \tau)=\left[T^{(1)^{*}}\left(\rho, \zeta, \tau-\tau_{k-1}\right)-2 T^{(1)^{*}}\left(\rho, \zeta, \tau-\tau_{k}\right)+T^{(1)^{*}}\left(\rho, \zeta, \tau-\tau_{k+1}\right),\right. \\
k=1,2, \ldots, n-1 \\
T_{n}^{(1)^{*}}(\rho, \zeta, \tau)=\left[T^{(1)^{*}}\left(\rho, \zeta, \tau-\tau_{n-1}\right)-T^{(1)^{*}}\left(\rho, \zeta, \tau-\tau_{n}\right)\right]-\left(\tau_{n}-\tau_{n-1}\right) T^{(0)^{*}}\left(\rho, \zeta, \tau-\tau_{n}\right),
\end{gathered}
$$

and dimensionless temperatures $T^{(0) *}$ and $T^{(1) *}$ can be derived from Eqs. (125) and (138) respectively.

Analogous quasi-static thermal stresses can be found as:

$$
\sigma_{i j}^{*}(\rho, \zeta, \tau)=\frac{1}{\delta \tau} \sum_{k=0}^{n} I\left(\tau_{k}\right) \sigma_{i j, k}^{(1)^{*}}(\rho, \zeta, \tau)
$$

where

$$
\begin{aligned}
& \sigma_{i j, 0}^{(1)^{*}}(\rho, \zeta, \tau)=\tau_{1} \sigma_{i j}^{(0)^{*}}(\rho, \zeta, \tau)-\sigma_{i j}^{(1)^{*}}(\rho, \zeta, \tau)+\sigma_{i j}^{(1)^{*}}\left(\rho, \zeta, \tau-\tau_{1}\right) \\
& \sigma_{i j, k}^{(1)^{*}}(\rho, \zeta, \tau)=\left[\sigma_{i j}^{(1)^{*}}\left(\rho, \zeta, \tau-\tau_{k-1}\right)-2 \sigma_{i j}^{(1)^{*}}\left(\rho, \zeta, \tau-\tau_{k}\right)+\sigma_{i j}^{(1)^{*}}\left(\rho, \zeta, \tau-\tau_{k+1}\right),\right. \\
& k=1,2, \ldots, n-1 \\
& \sigma_{i j, n}^{(1)^{*}}(\rho, \zeta, \tau)=\left[\sigma_{i j}^{(1)^{*}}\left(\rho, \zeta, \tau-\tau_{n-1}\right)-\sigma_{i j}^{(1)^{*}}\left(\rho, \zeta, \tau-\tau_{n}\right)\right]-\left(\tau_{n}-\tau_{n-1}\right) \sigma_{i j}^{(0)^{*}}\left(\rho, \zeta, \tau-\tau_{n}\right),
\end{aligned}
$$

and dimensionless thermal stresses $\sigma_{i j}^{(0)^{*}}$ and $\sigma_{i j}^{(1)^{*}}$ can be derived from Eqs. (127) and (139) respectively.

\subsection{Numerical analysis and conclusions}

Determination of non-stationary temperature fields and quasi-static thermal stresses fields were done for laser irradiation of semi-infinite surface of the body with the use of laser pulse shape described by the function $I(\tau)$. It was assumed the Poisson coefficient had value of $v=0,3$, and number of components in subtotals (145) and (151) was chosen from accuracy defined condition. Evolution of dimensionless temperature $T^{*}=T / T_{0}$ in defined points on the semi-infinite surface of the body $\zeta=0$ is shown on Fig. 17 and along symmetry axis $\rho=0$ on Fig. 18. 


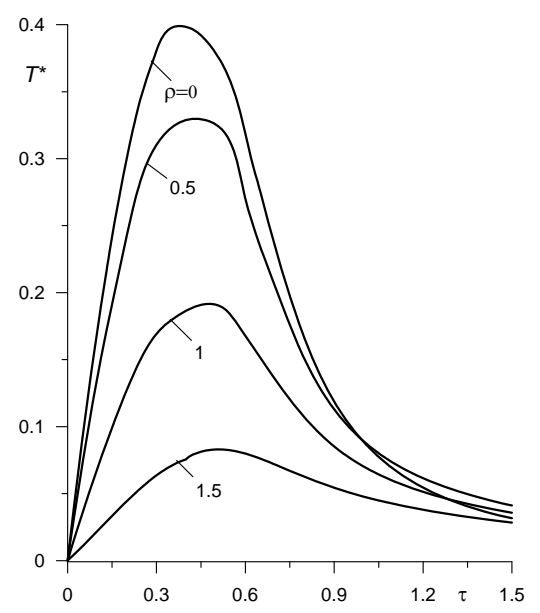

Fig. 17. Evolution of dimensionless temperature $T^{*}$ on the laser irradiated semi-infinite surface of the body $\zeta=0$ for different values of radial variable $\rho$ (Yevtushenko \& Matysiak, 2005).

Temperature in the centre of heated area $(\rho=0, \zeta=0)$ reaches maximum value at the moment $\tau_{r}=0.27$, when the laser irradiation intensity is the highest. After that, the cooling process begins as a result of decrease of laser irradiation intensity with time. With the distance from the heated centre area dimensionless time $\tau_{\max }$ of maximum temperature increases: for the values $\rho=0.5 ; 1 ; 1.5$ equals $\tau_{\max }=0.4 ; 0.48 ; 0.51$, respectively (see Fig. 17). Simultaneously with the dimensionless distance $\zeta$ from laser irradiated surface of the body, time of reaching the maximum temperature increases, too: for the values $\zeta=0.1 ; 0.25 ; 0.5$ equals $\tau_{\max }=0.1 ; 0.25 ; 0.5$, respectively (see Fig. 18). After switching laser system off $(\tau \geq 1)$, temperature along symmetry axis decreases to its starting value.

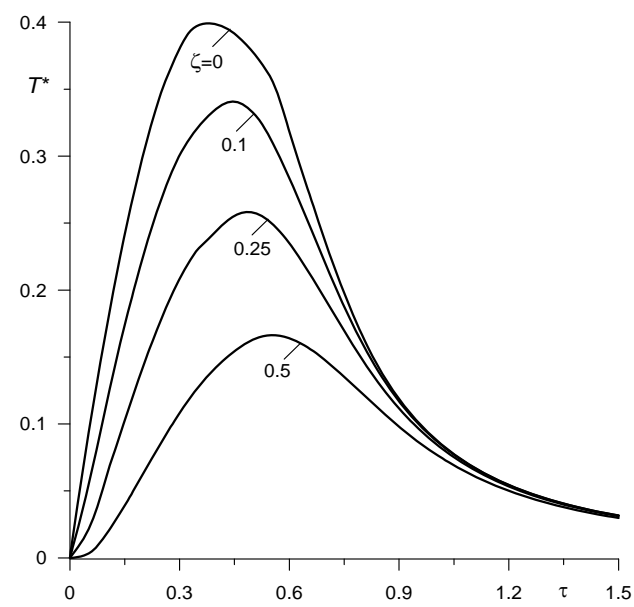

Fig. 18. Evolution of dimensionless temperature $T^{*}$ along symmetry axis $\rho=0$ for different values of dimensionless variable $\zeta$ (Yevtushenko \& Matysiak, 2005). 
Evolution with time of dimensionless thermal stresses $\sigma_{i j}^{*}=\sigma_{i j} / \sigma_{0}$ is shown on Fig. 19. Evolution of thermal radial stresses $\sigma_{r r}^{*}$ and peripheral $\sigma_{\theta \theta}^{*}$ in the chosen four points in the distance $\zeta=0.5$ from laser irradiated surface are very similar in nature (see Figs. 19, 20). Since switching the laser system on to the moment when $\tau_{r}=0.27$, stresses are stretching and afterward change their sign (become compressive one), then their absolute value significantly increases. Maximum value of these stresses is achieved on symmetry axis $\rho=0$ in time $\approx 2 \tau_{r}$.

In the starting moment of laser irradiation action, the dimensionless normal stresses $\sigma_{z z}^{*}$ is stretching but close to the moment of laser system switched off become compressive innature (see Fig. 21).

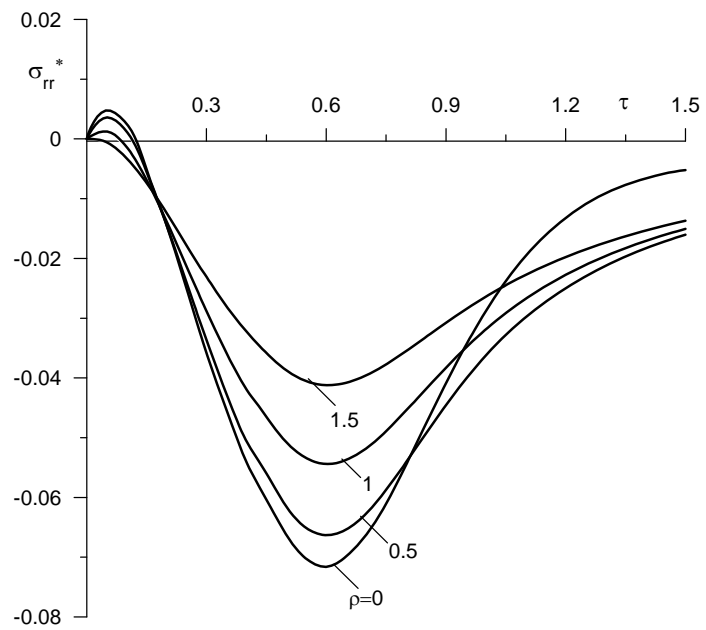

Fig. 19. Evolution of dimensionless thermal stresses $\sigma_{r r}^{*}$ inside the body $\zeta=0.5$ with the distance from the laser irradiated surface for different values of radial variable $\rho$ (Yevtushenko \& Matysiak, 2005).

At the moment when $\sigma_{z z}^{*}>0$, these stresses decrease with the distance from the symmetry axis. Appearance of the stretching and compressive normal stresses underneath the laser irradiated body surface can be explained by the thermal expansion of material in the period of irradiation intensity is increasing $0<\tau \leq 0.27$ and consequently by the compressing during the cooling process when $\tau>0.27$.

Dimensionless shear stresses $\sigma_{r z}^{*}$ are negative during almost all the heating interval and become positive after the laser system is switched off. It should be underlined that absolute value of shear stresses increases with the distance from symmetry axis $\rho=0$.

All the tensor components of stresses have insignificant values when $\tau>5$. Distribution of dimensionless radial stresses $\sigma_{r r}^{*}$ and normal $\sigma_{z z}^{*}$ along symmetry axis $\rho=0$ for different dimensionless time values is shown on Fig. 22, 23.

Maximum value of compressive stresses $\sigma_{r r}^{*}$ is reached when laser irradiation intensity is the highest ( $\tau=0.27$ ) (Fig. 22). With the distance from irradiated surface, larger then two radius of laser beam, the radial stresses negligibly small. Normal stresses $\sigma_{z z}^{*}$ equal zero on 
the laser irradiated surface $\zeta=0$ and increase with the distance from the semi-infinite surface of the body when finally reach some maximum value (see Fig. 23). These stresses are stretching when laser system is operating and become compressive when laser system is off.

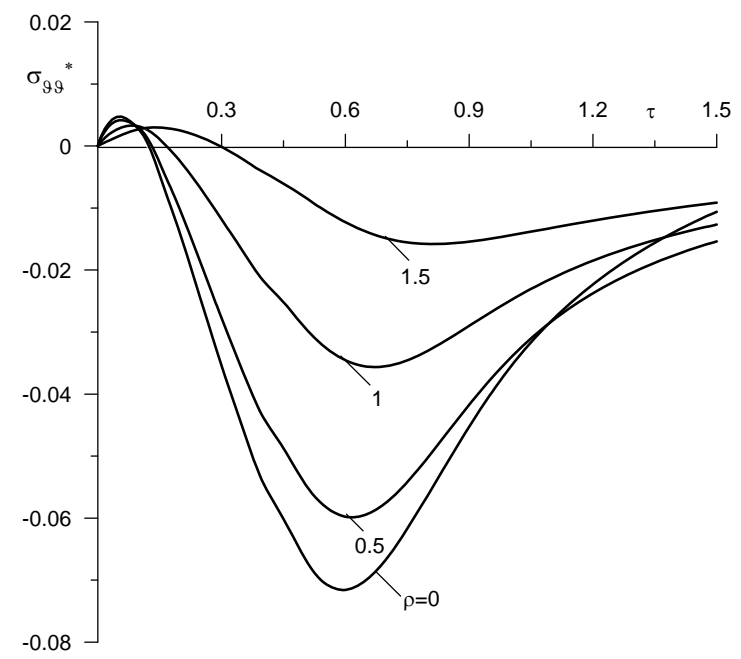

Fig. 20. Evolution of dimensionless thermal stresses $\sigma_{\theta \theta}^{*}$ inside the body $\zeta=0.5$ with the distance from the laser irradiated surface for different values of radial variable $\rho$ (Yevtushenko \& Matysiak, 2005).

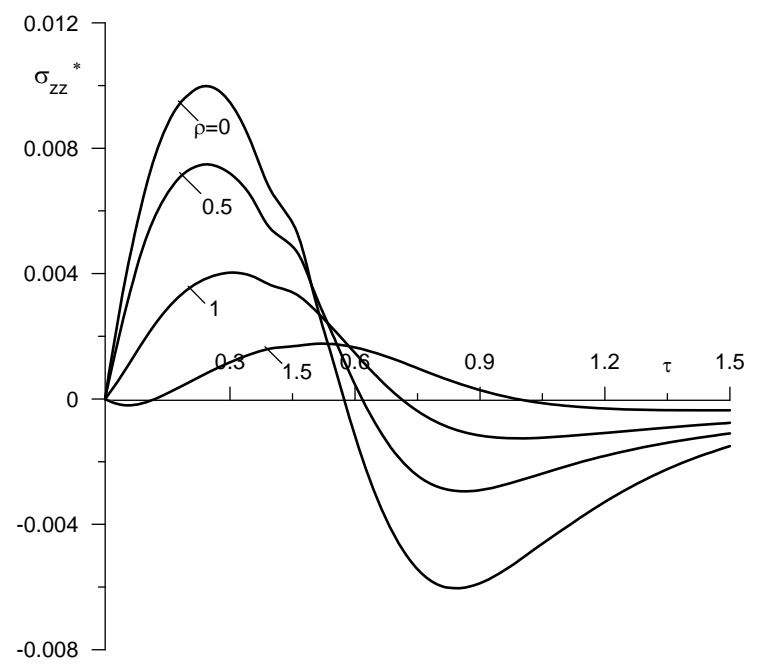

Fig. 21. Evolution of dimensionless thermal stresses $\sigma_{z z}^{*}$ inside the body $\zeta=0.5$ with the distance from the laser irradiated semi-infinite surface for different values of radial variable $\rho$ (Yevtushenko \& Matysiak, 2005). 


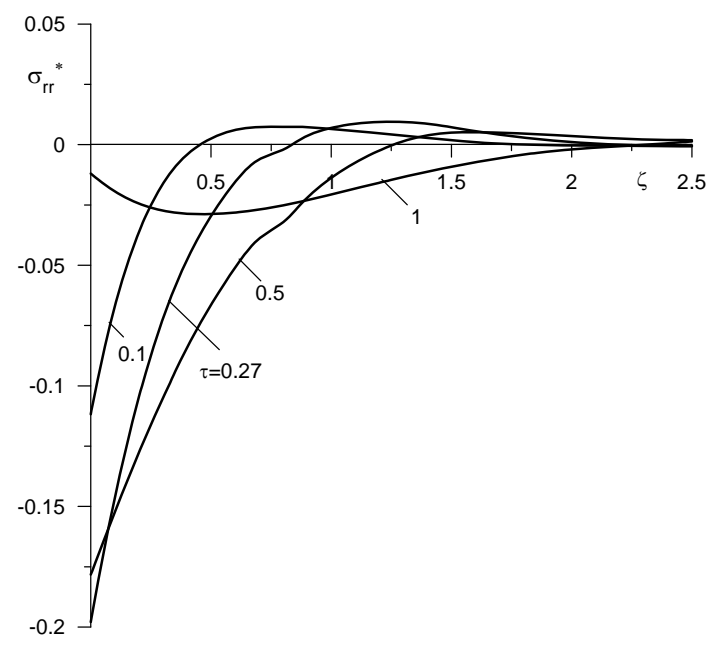

Fig. 22. Evolution of dimensionless thermal stresses $\sigma_{r r}^{*}$ along symmetry axis $\rho=0$ for different dimensionless time values (Yevtushenko \& Matysiak, 2005).

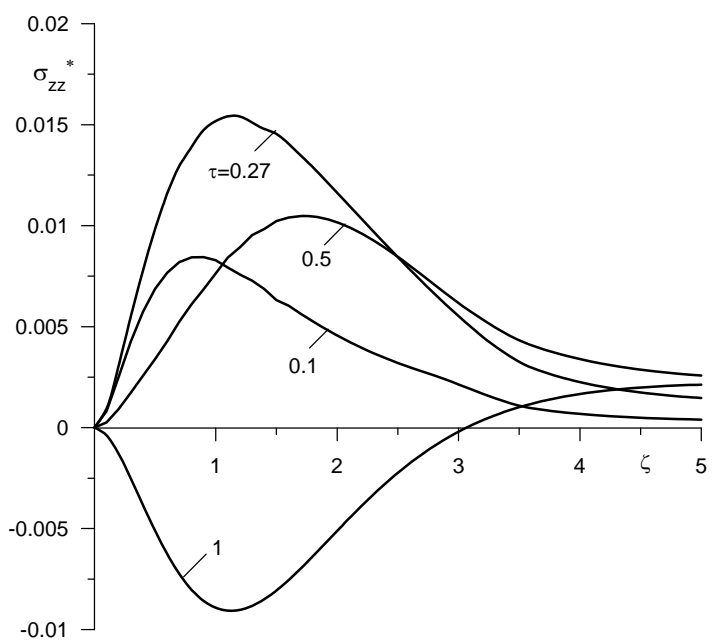

Fig. 23. Evolution of dimensionless thermal stresses $\sigma_{z z}^{*}$ along symmetry axis $\rho=0$ for different dimensionless time values (Yevtushenko \& Matysiak, 2005).

\section{References}

Abramowitz, M. \& Stegun, I.A. Handbook of Mathematical Functions with Formulas,

Graphs and Mathematical Tables, Wiley, New York, 1972, pp. 830.

Ashcroft, N. W. \& Mermin, N. D. Solid state physics, Warsaw: PWN, 1986. 
Aulyn, V. et al. Розвиток і використання макро- та мікроневрівноважних процесів у матеріалах при зміцненні й відновленні деталей лазерними технологіями, Mashinoznavstvo, 3 (2002), 31-37.

Bardybahin, A.I. \& Czubarov, Y.P. Influence of local irradiation intensity distribution in a plane normal to the laser beam axis on maximal temperature for the thin plate, Fizika i Chimia Obrabotki Materialov 4 (1996) 27-35.

Carslaw, H.S. \& Jaeger, J.C. Conduction of heat in solids, Oxford: 2nd ed. Clarendon Press, 1959.

Griffith A.A. The theory of rupture, Proc. 1-st Int. Congress of Appl. Mech., Delft, 1924, (Delft. Waltmar) 1926, p. 55.

Hector, L.G. \& Hetnarski, R.B. Thermal stresses in materials due to laser heating, in: R.B. Hetnarski, Thermal Stresses IV, Elsevier Science Publishers B.V., 1996, pp. 453-531.

Lauriello, P.J. \& Chen, Y. Thermal fracturing of hard rock, Trans. ASME. J. Appl. Mech., 1973, vol. 40, no. 4, p. 909.

Marchuk, G.I. \& Agoshkov, V.I. Introduction to Project-Mesh Methods (in Russian), Moskwa: Nauka, 1981.

Matysiak, S.J. et al. Temperature field in a microperiodic two-layered composite caused by a circular laser heat source, Heat Mass Tr., 1998, vol. 34, no. 1, p. 127.

McClintock F.A. \& Walsh J.B. Friction on Griffith cracks under pressure, Proc. 4-th U.S. Congress of Appl. Mech., Berkeley, 1962, p. 1015.

Nowacki, W. Thermoelasticity, Oxford: Pergamon Press, 1986.

Prudnikov, A.P. et al. Integrals and series. Vol. 2. Special Functions, New York-London: Taylor \& Francis, 1998, pp. 800.

Ready, J.F. Effects of high-power laser radiation, Academic Press, New York-London, 1971.

Rozniakowski, K. Application of laser radiation for examination and modification of building materials properties, BIGRAF, Warsaw, 2001, p. 198.

Rożniakowski, K. et al. Laser-induced temperature field and thermal stresses in the elstic homogeneous material, Materials Science, 2003, vol. 39, no. 3, p. 385-393.

Rykalin, N.N. et al. Laser processing of materials, (in Russian), Mashinostroenie, Moscow, 1975, pp. 296.

Sneddon, I.N. The use of integral transforms, New York: McGraw-Hill, 1972.

Timoshenko, S.P. \& Goodier, J.N. Theory of Elasticity, New York: McGraw-Hill, 1970.

Yevtushenko A.A. et al. Evaluation of effective absorption coefficient during laser irradiation using of metals martensite transformation, Heat Mass Tr., 2005, vol. 41, p. 338 .

Yevtushenko A.A. et al. Temperature and thermal stresses due to laser irradiation on construction materials (in Polish). Monograph, Bialystok: Technical University of Bialystok, 2009.

Yevtushenko, A.A. et al. On the modelling of laser thermal fracturing of hard rock, Engng. Trans., 1997, vol. 45 , no. 3/4, p. 447. 


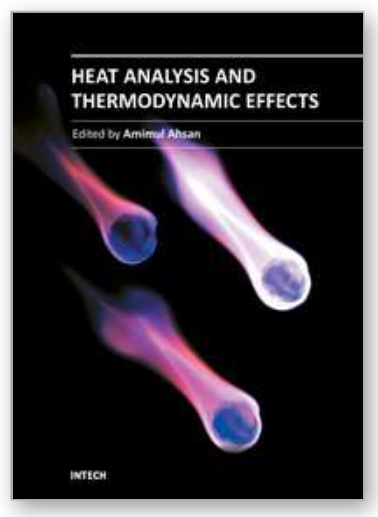

\author{
Heat Analysis and Thermodynamic Effects \\ Edited by Dr. Amimul Ahsan
}

ISBN 978-953-307-585-3

Hard cover, 394 pages

Publisher InTech

Published online 22, September, 2011

Published in print edition September, 2011

The heat transfer and analysis on heat pipe and exchanger, and thermal stress are significant issues in a design of wide range of industrial processes and devices. This book includes 17 advanced and revised contributions, and it covers mainly (1) thermodynamic effects and thermal stress, (2) heat pipe and exchanger, (3) gas flow and oxidation, and (4) heat analysis. The first section introduces spontaneous heat flow, thermodynamic effect of groundwater, stress on vertical cylindrical vessel, transient temperature fields, principles of thermoelectric conversion, and transformer performances. The second section covers thermosyphon heat pipe, shell and tube heat exchangers, heat transfer in bundles of transversely-finned tubes, fired heaters for petroleum refineries, and heat exchangers of irreversible power cycles. The third section includes gas flow over a cylinder, gas-solid flow applications, oxidation exposure, effects of buoyancy, and application of energy and thermal performance index on energy efficiency. The forth section presents integral transform and green function methods, micro capillary pumped loop, influence of polyisobutylene additions, synthesis of novel materials, and materials for electromagnetic launchers. The advanced ideas and information described here will be fruitful for the readers to find a sustainable solution in an industrialized society.

\title{
How to reference
}

In order to correctly reference this scholarly work, feel free to copy and paste the following:

Aleksander Yevtushenko, Kazimierz Rozniakowski and Malgorzata Rozniakowska-Klosinska (2011). AxiSymmetrical Transient Temperature Fields and Quasi-Static Thermal Stresses Initiated by a Laser Pulse in a Homogeneous Massive Body, Heat Analysis and Thermodynamic Effects, Dr. Amimul Ahsan (Ed.), ISBN: 978953-307-585-3, InTech, Available from: http://www.intechopen.com/books/heat-analysis-and-thermodynamiceffects/axi-symmetrical-transient-temperature-fields-and-quasi-static-thermal-stresses-initiated-by-a-laser-

\section{INTECH}

open science | open minds

\section{InTech Europe}

University Campus STeP Ri

Slavka Krautzeka 83/A

51000 Rijeka, Croatia

Phone: +385 (51) 770447

Fax: +385 (51) 686166

\section{InTech China}

Unit 405, Office Block, Hotel Equatorial Shanghai

No.65, Yan An Road (West), Shanghai, 200040, China 中国上海市延安西路65号上海国际贵都大饭店办公楼405单元

Phone: +86-21-62489820

Fax: $+86-21-62489821$ 
www.intechopen.com 
(C) 2011 The Author(s). Licensee IntechOpen. This chapter is distributed under the terms of the Creative Commons Attribution-NonCommercialShareAlike-3.0 License, which permits use, distribution and reproduction for non-commercial purposes, provided the original is properly cited and derivative works building on this content are distributed under the same license. 